\title{
On Wave Diffraction of Two-Dimensional Moonpools in a Two-Layer Fluid with Finite Depth
}

\author{
Xin $\mathrm{Xu}^{\mathrm{a}, \mathrm{b}}$, Xingyu Song ${ }^{\mathrm{a}, \mathrm{b}}$, Xinshu Zhang ${ }^{\mathrm{a}, \mathrm{b}, *}$, Zhiming Yuan ${ }^{\mathrm{c}, \mathrm{d}}$ \\ ${ }^{a}$ State Key Laboratory of Ocean Engineering, Shanghai Jiao Tong University, \\ Shanghai, 200240, China \\ ${ }^{b}$ Collaborative Innovation Center for Advanced Ship and Deep-Sea Exploration (CISSE), \\ Shanghai, 200240, China \\ ${ }^{c}$ Department of Naval Architecture, Ocean and Marine Engineering, University of Strathclyde, \\ 100 Montrose Street, Glasgow, G4 OLZ, UK \\ ${ }^{d}$ School of Naval Architecture and Ocean Engineering, Jiangsu University of Science and Technology, \\ Zhenjiang, 212003, China
}

\begin{abstract}
This paper studies the wave diffraction problem of two-dimensional moonpools in a two-layer fluid by using domain decomposition scheme and the method of eigenfunction expansion. Wave exciting forces, free surface and internal wave elevations are computed and analyzed for both surface wave and internal wave modes. The present model is validated by comparing a limiting case with a single-layer fluid case. Both piston mode and sloshing mode resonances have been identified and analyzed. It is observed that, compared with the solutions in surface wave mode, the wave exciting forces in internal wave mode are much smaller, and show more peaks and valleys in low-frequency region. As the wave frequency increases, the bandwidth of sloshing mode resonances decreases. Extensive parametric studies have been performed to examine the effects of moonpool geometry and density stratification on the resonant wave motion and exciting forces. It is found that, for twin bodies with deep draft in surface wave mode, the decreasing density ratio has little effects on the sloshing mode resonance frequencies but can somehow suppress the horizontal wave exciting forces and surface wave elevations around piston mode resonance region. In addition, the presence of lower-layer fluid can lead to the reduction of piston mode resonance frequency.
\end{abstract}

\section{Introduction}

Moonpool is an opening through the deck of the hull or offshore platform, giving passage to the drilling pipes, equipments and divers, etc. Two-dimensional moonpool can be adopted to model the gap between two ships moored in side-by-side arrangement, or the open area between the individual hulls of a multi-hull vessel. Exposed to incident waves or harmonic ship motions, the fluid inside moonpool domain can oscillate and suffer large-amplitude resonant motions. Hence, hydrodynamic analyses and optimization of moonpool structure are essential to the design and operation of ships or offshore structures.

Over the past few decades, moonpool resonance has received considerable attention. Molin (2001) and McIver (2005) developed a theoretical model based on linearized water wave theory to study the moonpool resonance. More recently, Molin et al. (2018) made an extension of the theoretical model to estimate the piston

\footnotetext{
* Corresponding author; Director, Innovative Marine Hydrodynamics Laboratory (iMHL).

Email address: xinshuz@sjtu.edu.cn (Xinshu Zhang)
} 
and sloshing mode resonance frequencies for moonpools in finite depth. The applications have been made for the cases of three-dimensional circular moonpool, rectangular gap and moonpool. Zhang et al. (2019) developed a theoretical model to predict the natural frequencies for two-dimensional asymmetric moonpool in finite water depth.

Yeung and Seah (2007) applied an eigenfunction matching method to analyze the Helmholtz resonance mode (also called piston mode) and higher-order symmetric sloshing modes of resonances for a moonpool formed by twin rectangular boxes in finite water depth. Faltinsen et al. (2007) studied the piston-like wave motion inside a two-dimensional moonpool based on the Galerkin method and compared the numerical solutions with the experimental data.

Kristiansen and Faltinsen (2008) adopted a vortex-tracking method and found that the flow separation at the bottom corner of the moonpool caused the discrepancies between the experimental measurements and the results using potential flow theory. Later, Faltinsen and Timokha (2015) investigated the problem by modifying the dynamic free surface condition inside moonpool domain through quantifying the pressure discharge to account for the damping caused by vortex shedding. Fredriksen et al. (2015) compared two numerical hybrid methods to investigate the resonant piston-mode motion in a moonpool and the rigid-body motions. Considering that the two bodies are not always symmetric, i.e. two vessels with different dimensions, Jiang et al. (2018) and Ning et al. (2018) investigated the surface wave elevation inside the moonpool formed by two rectangular boxes with different geometries.

Most of the previous studies are focused on the moonpool resonances in a single-layer fluid. However, stratified flow is also common in marine environment due to variation of water temperature and salinity with respect to the water depth (Osborne et al., 1977; Simmons et al., 2011; Xu et al., 2013). The pycnocline, separating two well-mixed fluid layers above and below, can be simply modeled by a two-layer fluid with constant density in each layer.

The behavior of the moonpool resonance in a two-layer fluid could be quite different from the scenario in a single-layer fluid. Yeung and Nguyen (1999) solved the wave radiation and diffraction problems for floating bodies in a two-layer fluid based on boundary integral equation method. Zhang and Bandyk (2013) studied the radiation problem for a symmetric moonpool in a two-layer fluid based on the method of eigenfunction matching. Later, this method was extended to study interface and surface-piercing twin bodies (Zhang and Bandyk, 2014). Recently, Zhang and Yeung (2016) studied the three-dimensional wave radiation problem of a cylindrical moonpool with an entrapped two-layer fluid. In short, there are still few literatures on moonpool resonance in a two-layer fluid, in particular for the wave diffraction problem.

The present paper aims to study the wave diffraction of a two-dimensional moonpool in a two-layer fluid with finite depth. A semi-analytical model is developed based on the domain decomposition and the method of eigenfunction expansion. The wave exciting forces on the twin bodies and wave elevations inside the moonpool are computed and analyzed. Both the piston mode and sloshing mode resonance behavior with physical interpretation are discussed with parametric studies being performed. 


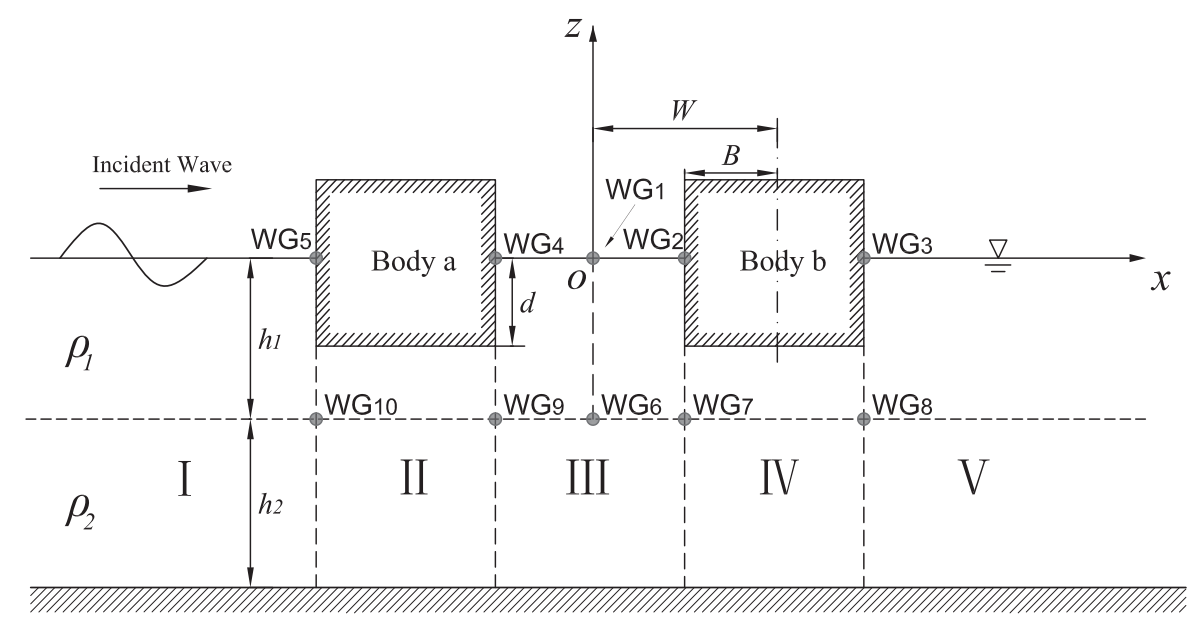

Figure 1: Sketch of the problem and coordinate system. WG denotes the numerical wave gauges placed to measure wave elevations. The coordinates of the wave gauges are: WG1 $(0,0)$, WG2 $(W-B, 0)$, WG3 $(W+B, 0)$, WG4 $(-W+B, 0)$, WG5 $(-W-B, 0)$, WG6 $\left(0,-h_{1}\right)$, WG7 $\left(W-B,-h_{1}\right)$, WG8 $\left(W+B,-h_{1}\right)$, WG9 $\left(-W+B,-h_{1}\right)$ and WG10 $\left(-W-B,-h_{1}\right)$.

\section{Mathematical formulation}

Wave diffraction by two rectangular boxes with identical geometry (twin bodies) in a two-layer fluid with finite depth are studied. The sketch of the problem is illustrated in Fig. 1. The densities of the upper-layer and lower-layer fluid are $\rho_{1}$ and $\rho_{2}$, respectively. The width of each rectangular body is $2 B$. The distance between the centers of the twin bodies is $2 W$. The draft of each floating box is $d$. The total fluid depth is $h$, including the upper-layer depth $h_{1}$ and the lower-layer depth $h_{2}$. The fluid is assumed to be incompressible and inviscid. A fixed Cartesian coordinate system oxz is defined with origin $o$ being located at the center of the moonpool. The $x$-axis is along the calm water free surface and the $z$-axis points upwards. Note that the present model only considers the case for $d<h_{1}$, and it is assumed the twin boxes do not penetrate the interfacial surface at $z=-h_{1}$.

The diffraction problem is considered in the present study as the incident wave propagates through the twin bodies. The velocity potential within the two-layer fluid can be written as

$$
\Phi^{(m)}=\operatorname{Re}\left\{\varphi^{(m)} e^{-i \omega t}\right\}
$$

where $\varphi^{(m)}$ are the spatial velocity potentials with $m=1,2$ representing the upper-layer and lower-layer fluid, respectively; $\omega$ is the angular frequency.

The velocity potentials of the incident wave with frequency $\omega$ can be written as

$$
\varphi_{0}^{(m)}(x, z)=\frac{g A_{p}}{i \omega} Z^{(m)}\left(k_{0}^{(p)}, z\right) e^{i k_{0}^{(p)} x},
$$

where $g$ is the gravitational acceleration; $A_{p}$ is related with the incoming wave amplitude, with $p=1,2$ representing the surface wave mode and the internal wave mode, respectively; $Z^{(m)}$ are the eigenfunctions in vertical direction; $k_{0}^{(p)}$ denote the wavenumbers corresponding to two incident propagating wave modes.

The values of $k_{n}^{(p)}$ are evaluated based on the dispersion relations which can be derived by means of separation of variables. 
For $n=0$ :

$$
\frac{\omega^{2}}{g}=\frac{k_{0}^{(p)}}{2\left(1+\gamma t_{1} t_{2}\right)}\left\{t_{1}+t_{2}+(-1)^{p+1} \cdot \sqrt{\left(t_{1}+t_{2}\right)^{2}-4 \varepsilon t_{1} t_{2}\left(1+\gamma t_{1} t_{2}\right)}\right\},
$$

with density ratio $\gamma=\rho_{1} / \rho_{2}, \varepsilon=1-\gamma, t_{1}=\tanh k_{0}^{(p)} h_{1}, t_{2}=\tanh k_{0}^{(p)} h_{2}$. Yeung and Nguyen (1999) derived the dispersion relation given in Eqn. (3) for outgoing waves.

For $n>0$ :

$$
\frac{\omega^{2}}{g}=\frac{-k_{n}^{(p)}}{2\left(1-\gamma t_{1} t_{2}\right)}\left\{t_{1}+t_{2}+(-1)^{p+1} \cdot \sqrt{\left(t_{1}+t_{2}\right)^{2}-4 \varepsilon t_{1} t_{2}\left(1-\gamma t_{1} t_{2}\right)}\right\},
$$

with $t_{1}=\tan k_{n}^{(p)} h_{1}, t_{2}=\tan k_{n}^{(p)} h_{2}$. Zhang and Bandyk (2013) gave the dispersion relation given in Eqn. (4) for evanescent modes.

For either surface incident wave mode or internal incident wave mode, the scattered waves consist of two modes. The velocity potentials of diffracted waves consist of both the surface wave mode $\left(k_{0}^{(1)}\right)$ and the internal wave mode $\left(k_{0}^{(2)}\right)$. The wavenumbers $k_{0}^{(q)}(q=1,2)$, corresponding to the outgoing wave, satisfy the dispersion relation Eqn. (3), while wavenumbers $k_{n}^{(q)}(q=1,2 ; n=1,2, \ldots, \infty)$, corresponding to the evanescent modes, satisfy the dispersion relation Eqn. (4).

The spatial velocity potential should satisfy the Laplace equation

$$
\left(\frac{\partial^{2}}{\partial x^{2}}+\frac{\partial^{2}}{\partial z^{2}}\right) \varphi^{(m)}=0
$$

The linearized free surface boundary condition is written as

$$
\varphi_{z}^{(1)}-K \varphi^{(1)}=0, \quad \text { at } z=0
$$

where $K=\omega^{2} / g$.

The boundary conditions at seabed and the bottom of the moonpool are written as

$$
\begin{aligned}
& \varphi_{z}^{(2)}=0, \quad \text { at } z=-h, \\
& \varphi_{z}^{(1)}=0, \quad \text { at } z=-d .
\end{aligned}
$$

The vertical velocity and linear hydrodynamic pressure at the interfacial surface are continuous

$$
\begin{gathered}
\varphi_{z}^{(2)}=\varphi_{z}^{(1)}, \quad \text { at } z=-h_{1} \\
\gamma\left(\varphi_{z}^{(1)}-K \varphi^{(1)}\right)=\varphi_{z}^{(2)}-K \varphi^{(2)}, \quad \text { at } z=-h_{1} .
\end{gathered}
$$

There is no horizontal velocity on the body surfaces, so that the body boundary conditions are written as

$$
\begin{array}{ll}
\left.\varphi_{x}^{(1)}\right|_{x=\mp(W+B)}=0, & -d \leq z \leq 0, \\
\left.\varphi_{x}^{(1)}\right|_{x=\mp(W-B)}=0, & -d \leq z \leq 0 .
\end{array}
$$




\subsection{Domain decomposition}

The whole fluid domain is divided into five subdomains I (for $x \leq-W-B$ ), II (for $-W-B \leq x \leq B-W$ ), III (for $B-W \leq x \leq W-B$ ), IV (for $W-B \leq x \leq W+B$ ), and $\mathrm{V}$ (for $x \geq W+B$ ), as illustrated in Fig. 1. The velocity potentials and the horizontal velocities of two adjacent subdomains should be matched at the common boundaries, and can be written as follow

$$
\begin{aligned}
& I-I I:\left\{\begin{array}{l}
\varphi_{I}=\varphi_{I I} \\
\frac{\partial \varphi_{I}}{\partial x}=\frac{\partial \varphi_{I I}}{\partial x}
\end{array} \quad, \quad-h \leq z \leq-d, x=-W-B,\right. \\
& I I-I I I:\left\{\begin{array}{l}
\varphi_{I I}=\varphi_{I I I} \\
\frac{\partial \varphi_{I I}}{\partial x}=\frac{\partial \varphi_{I I I}}{\partial x}
\end{array} \quad, \quad-h \leq z \leq-d, x=-W+B\right. \\
& I I I-I V:\left\{\begin{array}{l}
\varphi_{I I I}=\varphi_{I V} \\
\frac{\partial \varphi_{I I I}}{\partial x}=\frac{\partial \varphi_{I V}}{\partial x}
\end{array} \quad, \quad-h \leq z \leq-d, x=W-B\right. \\
& I V-V:\left\{\begin{array}{l}
\varphi_{I V}=\varphi_{V} \\
\frac{\partial \varphi_{I V}}{\partial x}=\frac{\partial \varphi_{V}}{\partial x}
\end{array} \quad, \quad-h \leq z \leq-d, x=W+B .\right.
\end{aligned}
$$

\subsection{Solution in subdomain I}

By applying the method of separation of variables, the velocity potentials in subdomain I can be written as

$$
\begin{aligned}
\varphi_{I}^{(m)} & =\frac{A_{p} g}{i \omega}\left\{C_{1,0}^{I} Z^{(m)}\left(k_{0}^{(1)}, z\right) e^{-i k_{0}^{(1)}(x+W+B)}+C_{2,0}^{I} Z^{(m)}\left(k_{0}^{(2)}, z\right) e^{-i k_{0}^{(2)}(x+W+B)}\right\} \\
& +\frac{A_{p} g}{i \omega}\left\{\sum_{n=1}^{\infty} C_{1, n}^{I} Z^{(m)}\left(k_{n}^{(1)}, z\right) e^{k_{n}^{(1)}(x+W+B)}+\sum_{n=1}^{\infty} C_{2, n}^{I} Z^{(m)}\left(k_{n}^{(2)}, z\right) e^{k_{n}^{(2)}(x+W+B)}\right\} \\
& +\frac{g A_{p}}{i \omega} Z^{(m)}\left(k_{0}^{(p)}, z\right) e^{i k_{0}^{(p)} x},
\end{aligned}
$$

where $m=1,2$ represent the upper-layer and lower-layer fluid, respectively; $p=1,2$ denote the surface wave mode and the internal wave mode, respectively; $A_{p}(p=1,2)$ are the known coefficients related with the incident wave amplitude; $C_{q, n}^{I}(q=1,2 ; n=0,1, \ldots, \infty)$ are the unknown coefficients which will be determined by matching the velocities and the velocity potentials at the common boundary between subdomains I and II; $k_{n}^{(p)}$ are the eigenvalues defined by the dispersion relations $(3)$ and $(4) ; Z^{(m)}$ are the eigenfunctions in the vertical direction computed by Eqns. (18) and (19). It should be noted that the eigenvalues and eigenfunctions are the same for subdomains I, III and V.

For $n=0$ :

For $n \geq 1$ :

$$
Z^{(m)}\left(k_{0}^{(p)}, z\right)= \begin{cases}\frac{\frac{\omega^{2}}{g k_{0}^{(p)}} \sinh k_{0}^{(p)} z+\cosh k_{0}^{(p)} z}{\alpha\left(k_{0}^{(p)}\right)}, & m=1, \\ \frac{\omega^{2} \cosh k_{0}^{(p)}(z+h)}{g k_{0}^{(p)} \sinh k_{0}^{(p)} h_{2}}, & m=2 .\end{cases}
$$

$$
Z^{(m)}\left(k_{n}^{(p)}, z\right)= \begin{cases}\frac{\frac{\omega^{2}}{g k_{n}^{(p)}} \sin k_{n}^{(p)} z+\cos k_{n}^{(p)} z}{\alpha\left(k_{n}^{(p)}\right)}, & m=1, \\ \frac{\omega^{2} \cos k_{n}^{(p)}(z+h)}{g k_{n}^{(p)} \sin k_{n}^{(p)} h_{2}}, & m=2,\end{cases}
$$


where the amplitude ratio $\alpha\left(k_{n}^{(p)}\right)$ are defined by

$$
\alpha\left(k_{n}^{(p)}\right)= \begin{cases}\cosh k_{0}^{(p)} h_{1}\left(1-\frac{g k_{0}^{(p)}}{\omega^{2}} \tanh k_{0}^{(p)} h_{1}\right), & n=0, \\ \cos k_{n}^{(p)} h_{1}\left(-1-\frac{g k_{n}^{(p)}}{\omega^{2}} \tan k_{n}^{(p)} h_{1}\right), & n \geq 1 .\end{cases}
$$

Since the eigenfunctions $Z^{(m)}\left(k_{n}^{(p)}, z\right)$ are orthonormal sets, the following inner product can be defined for any combination of non-negative integers $n$ and $f$.

$$
\left\langle Z^{(m)}\left(k_{n}^{(p)}, z\right), Z^{(m)}\left(k_{f}^{(p)}, z\right)\right\rangle \equiv \frac{1}{h} \int_{-h}^{0} w(z) Z^{(m)}\left(k_{n}^{(p)}, z\right) Z^{(m)}\left(k_{f}^{(p)}, z\right) d z=\delta_{n, f} M_{n}^{(p)},
$$

where $\delta_{n, f}$ is the Kronecker delta and $M_{n}^{(p)}$ are defined as

$$
M_{n}^{(p)}=\frac{1}{h} \int_{-h}^{0} w(z) Z^{(m)}\left(k_{n}^{(p)}, z\right) Z^{(m)}\left(k_{n}^{(p)}, z\right) d z,
$$

with

$$
w(z)=\left\{\begin{array}{l}
\gamma,-h_{1} \leq z \leq 0, \\
1,-h \leq z \leq-h_{1} .
\end{array}\right.
$$

The coefficients $A_{p}$ are given as follows

$$
A_{p}= \begin{cases}a_{1} \alpha\left(k_{0}^{(1)}\right), & p=1, \\ a_{2}, & p=2,\end{cases}
$$

where $a_{1}$ and $a_{2}$ are the amplitudes of incident surface wave $(p=1)$ and incident internal wave $(p=2)$, respectively.

It should be noted that the two terms in the first brace in Eqn. (17) represent outgoing waves in surface and internal wave modes, respectively. The two terms in the second brace in Eqn. (17) denote the evanescent modes due to diffracted surface and internal waves, respectively. The last term in Eqn. (17) represents the incident wave.

\subsection{Solution in subdomain II}

By applying the method of separation of variables, the velocity potentials in subdomain II can be written as

$$
\begin{aligned}
\varphi_{I I}^{(m)} & =\frac{g}{i \omega} Z_{I I}^{(m)}\left(\lambda_{0}, z\right)\left[C_{0}^{I I}+D_{0}^{I I} \frac{x+W}{B}\right]+\frac{g}{i \omega} Z_{I I}^{(m)}\left(\lambda_{1}, z\right)\left[C_{1}^{I I} \frac{\cos \lambda_{1}(x+W)}{\cos \lambda_{1} B}+D_{1}^{I I} \frac{\sin \lambda_{1}(x+W)}{\sin \lambda_{1} B}\right] \\
& +\frac{g}{i \omega} \sum_{j=2}^{\infty} Z_{I I}^{(m)}\left(\lambda_{j}, z\right)\left[C_{j}^{I I} \frac{\cosh \lambda_{j}(x+W)}{\cosh \lambda_{j} B}+D_{j}^{I I} \frac{\sinh \lambda_{j}(x+W)}{\sinh \lambda_{j} B}\right],
\end{aligned}
$$

where $m=1,2$ represent the upper-layer and lower-layer fluid, respectively; $C_{j}^{I I}$ and $D_{j}^{I I}(j=0,1, \ldots, \infty)$ are the unknown coefficients which will be determined by matching the velocities and the velocity potentials at the boundary between subdomains I and II, and at the boundary between subdomains II and III; $Z_{I I}^{(m)}$ are the eigenfunctions in the vertical direction computed by Eqns. (26), (27) and (28). It should be noted that the eigenvalues and eigenfunctions are the same for subdomains II and IV. 
For $j=0$ :

$$
Z_{I I}^{(m)}= \begin{cases}1, & m=1 \\ \gamma, & m=2\end{cases}
$$

For $j=1$ :

$$
Z_{I I}^{(m)}= \begin{cases}\frac{\cosh \lambda_{1}(z+d)}{\sinh \lambda_{1}\left(d-h_{1}\right)}, & m=1 \\ \frac{\cosh \lambda_{1}(z+h)}{\sinh \lambda_{1} h_{2}}, & m=2 .\end{cases}
$$

For $j \geq 2$ :

$$
Z_{I I}^{(m)}= \begin{cases}\frac{\cos \lambda_{j}(z+d)}{\sin \lambda_{j}\left(d-h_{1}\right)}, & m=1, \\ \frac{\cos \lambda_{j}(z+h)}{\sin \lambda_{j} h_{2}}, & m=2,\end{cases}
$$

where the eigenvalues $\lambda_{j}$ are defined as

$$
\begin{cases}\lambda_{0}=0, & j=0 \\ \omega^{2}=\frac{(1-\gamma) g \lambda_{1}}{\gamma T_{1}+T_{2}}, & j=1 \\ \omega^{2}=\frac{-(1-\gamma) g \lambda_{j}}{\gamma T_{1}+T_{2}}, & j \geq 2\end{cases}
$$

with

$$
\begin{aligned}
& T_{1}\left(\lambda_{j}\right)=\left\{\begin{array}{cc}
\operatorname{coth} \lambda_{j}\left(h_{1}-d\right), & j=1, \\
\cot \lambda_{j}\left(h_{1}-d\right), & j \geq 2,
\end{array}\right. \\
& T_{2}\left(\lambda_{j}\right)=\left\{\begin{array}{cc}
\operatorname{coth} \lambda_{j} h_{2}, \quad j=1, \\
\cot \lambda_{j} h_{2}, \quad j \geq 2 .
\end{array}\right.
\end{aligned}
$$

Since the eigenfunctions $Z_{I I}^{(m)}\left(\lambda_{j}, z\right)$ are orthonormal sets, the following inner product can be defined for any combination of non-negative integers $e$ and $j$.

$$
\left\langle Z_{I I}^{(m)}\left(\lambda_{e}, z\right), Z_{I I}^{(m)}\left(\lambda_{j}, z\right)\right\rangle \equiv \frac{1}{h-d} \int_{-h}^{-d} w(z) Z_{I I}^{(m)}\left(\lambda_{e}, z\right) Z_{I I}^{(m)}\left(\lambda_{j}, z\right) d z=\delta_{e, j} N_{j}
$$

where $N_{j}$ is defined as

$$
N_{j}=\frac{1}{h-d} \int_{-h}^{-d} w(z) Z_{I I}^{(m)}\left(\lambda_{j}, z\right) Z_{I I}^{(m)}\left(\lambda_{j}, z\right) d z
$$

\subsection{Solution in subdomain III}

By applying the method of separation of variables, the velocity potentials in subdomain III can be written

as

$$
\begin{aligned}
\varphi_{I I I}^{(m)} & =\frac{g A_{p}}{i \omega} Z^{(m)}\left(k_{0}^{(1)}, z\right)\left[C_{1,0}^{I I I} \frac{\cos k_{0}^{(1)} x}{\cos k_{0}^{(1)}(W-B)}+D_{1,0}^{I I I} \frac{\sin k_{0}^{(1)} x}{\sin k_{0}^{(1)}(W-B)}\right] \\
& +\sum_{n=1}^{\infty} \frac{g A_{p}}{i \omega} Z^{(m)}\left(k_{n}^{(1)}, z\right)\left[C_{1, n}^{I I I} \frac{\cosh k_{n}^{(1)} x}{\cosh k_{n}^{(1)}(W-B)}+D_{1, n}^{I I I} \frac{\sinh k_{n}^{(1)} x}{\sinh k_{n}^{(1)}(W-B)}\right] \\
& +\frac{g A_{p}}{i \omega} Z^{(m)}\left(k_{0}^{(2)}, z\right)\left[C_{2,0}^{I I I} \frac{\cos k_{0}^{(2)} x}{\cos k_{0}^{(2)}(W-B)}+D_{2,0}^{I I I} \frac{\sin k_{0}^{(2)} x}{\sin k_{0}^{(2)}(W-B)}\right] \\
& +\sum_{n=1}^{\infty} \frac{g A_{p}}{i \omega} Z^{(m)}\left(k_{n}^{(2)}, z\right)\left[C_{2, n}^{I I I} \frac{\cosh k_{n}^{(2)} x}{\cosh k_{n}^{(2)}(W-B)}+D_{2, n}^{I I I} \frac{\sinh k_{n}^{(2)} x}{\sinh k_{n}^{(2)}(W-B)}\right],
\end{aligned}
$$


where $C_{q, n}^{I I I}$ and $D_{q, n}^{I I I}(q=1,2 ; n=0,1, \ldots, \infty)$ are the unknown coefficients which will be determined by matching the horizontal velocities and the velocity potentials at the juncture boundary shared by subdomains II and III, and at the common boundary between subdomains III and IV; $Z^{(m)}$ are the eigenfunctions in the vertical direction and can be computed by Eqns. (18) and (19).

\subsection{Solution in subdomain $I V$}

By applying the method of separation of variables, the velocity potentials in subdomain IV can be written as

$$
\begin{aligned}
\varphi_{I V}^{(m)} & =\frac{g}{i \omega} Z_{I I}^{(m)}\left(\lambda_{0}, z\right)\left[C_{0}^{I V}+D_{0}^{I V} \frac{x-W}{B}\right]+\frac{g}{i \omega} Z_{I I}^{(m)}\left(\lambda_{1}, z\right)\left[C_{1}^{I V} \frac{\cos \lambda_{1}(x-W)}{\cos \lambda_{1} B}+D_{1}^{I V} \frac{\sin \lambda_{1}(x-W)}{\sin \lambda_{1} B}\right] \\
& +\frac{g}{i \omega} \sum_{j=2}^{\infty} Z_{I I}^{(m)}\left(\lambda_{j}, z\right)\left[C_{j}^{I V} \frac{\cosh \lambda_{j}(x-W)}{\cosh \lambda_{j} B}+D_{j}^{I V} \frac{\sinh \lambda_{j}(x-W)}{\sinh \lambda_{j} B}\right],
\end{aligned}
$$

where $C_{j}^{I V}$ and $D_{j}^{I V}(j=0,1, \ldots, \infty)$ are the unknown coefficients which will be determined by matching the velocities and the velocity potentials at the boundary between subdomains III and IV, and at the boundary between subdomains IV and V.

\subsection{Solution in subdomain $V$}

By applying the method of separation of variables, the velocity potentials in subdomain $\mathrm{V}$ can be written as

$$
\begin{aligned}
\varphi_{V}^{(m)} & =\frac{g A_{p} C_{1,0}^{V}}{i \omega} Z^{(m)}\left(k_{0}^{(1)}, z\right) e^{i k_{0}^{(1)}(x-W-B)}+\frac{g A_{p} C_{2,0}^{V}}{i \omega} Z^{(m)}\left(k_{0}^{(2)}, z\right) e^{i k_{0}^{(2)}(x-W-B)} \\
& +\sum_{n=1}^{\infty} \frac{g A_{p} C_{1, n}^{V}}{i \omega} Z^{(m)}\left(k_{n}^{(1)}, z\right) e^{-k_{n}^{(1)}(x-W-B)}+\sum_{n=1}^{\infty} \frac{g A_{p} C_{2, n}^{V}}{i \omega} Z^{(m)}\left(k_{n}^{(2)}, z\right) e^{-k_{n}^{(2)}(x-W-B)},
\end{aligned}
$$

where $C_{q, n}^{V}(q=1,2 ; n=0,1, \ldots, \infty)$ are the unknown coefficients which will be determined by matching the velocities and the velocity potentials at the boundary between subdomains IV and V.

\subsection{Matching of solutions in the subdomains}

The expansions of $\varphi_{I}$ and $\varphi_{I I}$ can be introduced into the first equation in (13) to fulfill the matching boundary condition of velocity potentials at $x=-W-B$. Facilitated by using the orthonormal condition (31), it results in

$$
C_{j}^{I I}-D_{j}^{I I}=\frac{A_{p}}{N_{j}}\left\{e^{s_{0}^{(p)}(W+B)} L_{j, 0}^{(1)}+\sum_{n=0}^{\infty} C_{1, n}^{I} L_{j, n}^{(1)}+\sum_{n=0}^{\infty} C_{2, n}^{I} L_{j, n}^{(2)}\right\}, j=0,1, \ldots, \infty,
$$

with

$$
\begin{gathered}
s_{0}^{(p)}=-i k_{0}^{(p)}, \\
L_{j, n}^{(q)}=\int_{-h}^{-d} w(z) Z_{I I}^{(m)} Z^{(m)} d z .
\end{gathered}
$$

For $n \geq 1, s_{n}^{(p)}=k_{n}^{(p)}$. It should be noted that $s_{n}^{(q)}(n \geq 0)$ share the same definition as $s_{n}^{(p)}$. 
By substituting $\varphi_{I}$ and $\varphi_{I I}$ into the second equation in (13), the horizontal velocities in subdomains I and II can be matched at $x=-W-B$. After applying the orthonormal condition (21), it yields, for $p=q$ and $n=0$

$$
\begin{aligned}
s_{0}^{(q)} A_{p}\left(C_{q, 0}^{I}-e^{s_{0}^{(q)}}(W+B)\right) & =\frac{D_{0}^{I I}}{B} \frac{L_{0,0}^{(q)}}{M_{0}^{(q)}}+\left(C_{1}^{I I} \lambda_{1} \tan \lambda_{1} B+\frac{D_{1}^{I I} \lambda_{1}}{\tan \lambda_{1} B}\right) \frac{L_{1,0}^{(q)}}{M_{0}^{(q)}} \\
& +\sum_{j=2}^{\infty} \lambda_{j}\left(-C_{j}^{I I} \tanh \lambda_{j} B+\frac{D_{j}^{I I}}{\tanh \lambda_{j} B}\right) \frac{L_{j, 0}^{(q)}}{M_{0}^{(q)}}
\end{aligned}
$$

for the other conditions

$$
\begin{aligned}
C_{q, n}^{I} A_{p} s_{n}^{(q)} & =\frac{D_{0}^{I I}}{B} \frac{L_{0, n}^{(q)}}{M_{n}^{(q)}}+\left(C_{1}^{I I} \lambda_{1} \tan \lambda_{1} B+\frac{D_{1}^{I I} \lambda_{1}}{\tan \lambda_{1} B}\right) \frac{L_{1, n}^{(q)}}{M_{n}^{(q)}} \\
& +\sum_{j=2}^{\infty} \lambda_{j}\left(-C_{j}^{I I} \tanh \lambda_{j} B+\frac{D_{j}^{I I}}{\tanh \lambda_{j} B}\right) \frac{L_{j, n}^{(q)}}{M_{n}^{(q)}}, n=1,2, \ldots, \infty .
\end{aligned}
$$

The expansions of $\varphi_{I I}$ and $\varphi_{I I I}$ can be introduced into the first equation in (14) to fulfill the matching boundary condition of velocity potentials at $x=-W+B$. By using the orthonormal condition (31), it results in

$$
C_{j}^{I I}+D_{j}^{I I}=\frac{A_{p}}{N_{j}}\left[\sum_{n=0}^{\infty} L_{j, n}^{(1)}\left(C_{1, n}^{I I I}-D_{1, n}^{I I I}\right)+\sum_{n=0}^{\infty} L_{j, n}^{(2)}\left(C_{2, n}^{I I I}-D_{2, n}^{I I I}\right)\right], j=0,1, \ldots, \infty .
$$

By substituting $\varphi_{I I}$ and $\varphi_{I I I}$ into the second equation in (14), the horizontal velocities in subdomains II and III can be matched at $x=-W+B$. After applying the orthonormal condition (21), it yields

$$
\begin{aligned}
& A_{p} s_{n}^{(q)}\left[-C_{q, n}^{I I I} \tanh s_{n}^{(q)}(W-B)+\frac{D_{q, n}^{I I I}}{\tanh s_{n}^{(q)}(W-B)}\right] \\
& =\frac{D_{0}^{I I}}{B} \frac{L_{0, n}^{(q)}}{M_{n}^{(q)}}-\left(C_{1}^{I I} \lambda_{1} \tan \lambda_{1} B-\frac{D_{1}^{I I} \lambda_{1}}{\tan \lambda_{1} B}\right) \frac{L_{1, n}^{(q)}}{M_{n}^{(q)}} \\
& +\sum_{j=2}^{\infty} \frac{L_{j, n}^{(q)}}{M_{n}^{(q)}} \lambda_{j}\left(C_{j}^{I I} \tanh \lambda_{j} B+\frac{D_{j}^{I I}}{\tanh \lambda_{j} B}\right), n=0,1, \ldots, \infty .
\end{aligned}
$$

By substituting $\varphi_{I I I}$ and $\varphi_{I V}$ into the first equation in (15), the velocity potentials in subdomains III and IV can be matched at $x=W-B$. After applying the orthonormal condition for $Z_{I V}^{(m)}$, it yields

$$
C_{j}^{I V}-D_{j}^{I V}=\frac{A_{p}}{N_{j}}\left[\sum_{n=0}^{\infty} L_{j, n}^{(1)}\left(C_{1, n}^{I I I}+D_{1, n}^{I I I}\right)+\sum_{n=0}^{\infty} L_{j, n}^{(2)}\left(C_{2, n}^{I I I}+D_{2, n}^{I I I}\right)\right], j=0,1, \ldots, \infty .
$$

By substituting $\varphi_{I I I}$ and $\varphi_{I V}$ into the second equation in (15), the horizontal velocities in subdomains III and IV can be matched at $x=W-B$. After applying the orthonormal condition (21), it yields

$$
\begin{aligned}
& A_{p} s_{n}^{(q)}\left(C_{q, n}^{I I I} \tanh s_{n}^{(q)}(W-B)+\frac{D_{q, n}^{I I}}{\tanh s_{n}^{(q)}(W-B)}\right) \\
& =\frac{D_{0}^{I V}}{B} \frac{L_{0, n}^{(q)}}{M_{n}^{(q)}}+\left(C_{1}^{I V} \lambda_{1} \tan \lambda_{1} B+\frac{D_{1}^{I V} \lambda_{1}}{\tan \lambda_{1} B}\right) \frac{L_{1, n}^{(q)}}{M_{n}^{(q)}} \\
& +\sum_{j=2}^{\infty} \frac{L_{j, n}^{(q)}}{M_{n}^{(q)}} \lambda_{j}\left(-C_{j}^{I V} \tanh \lambda_{j} B+\frac{D_{j}^{I V}}{\tanh \lambda_{j} B}\right), n=0,1, \ldots, \infty .
\end{aligned}
$$


By substituting $\varphi_{I V}$ and $\varphi_{V}$ into the first equation in (16), the velocity potentials in subdomains IV and $\mathrm{V}$ can be matched at $x=W+B$. After applying the orthonormal condition for $Z_{I V}^{(m)}$, it gives

$$
C_{j}^{I V}+D_{j}^{I V}=\frac{A_{p}}{N_{j}}\left[\sum_{n=0}^{\infty} C_{1, n}^{V} L_{j, n}^{(1)}+\sum_{n=0}^{\infty} C_{2, n}^{V} L_{j, n}^{(2)}\right], j=0,1, \ldots, \infty .
$$

By substituting $\varphi_{I V}$ and $\varphi_{V}$ into the second equation in (16), the horizontal velocities in subdomains IV and $\mathrm{V}$ can be matched at $x=W+B$. After applying the orthonormal condition (21), it yields

$$
\begin{aligned}
-A_{p} C_{q, n}^{V} s_{n}^{(q)} & =\frac{D_{0}^{I V}}{B} \frac{L_{0, n}^{(q)}}{M_{n}^{(q)}}+\left(-C_{1}^{I V} \lambda_{1} \tan \lambda_{1} B+\frac{D_{1}^{I V} \lambda_{1}}{\tan \lambda_{1} B}\right) \frac{L_{1, n}^{(q)}}{M_{n}^{(q)}} \\
& +\sum_{j=2}^{\infty} \frac{L_{j, n}^{(q)}}{M_{n}^{(q)}} \lambda_{j}\left(C_{0}^{I V} \tanh \lambda_{j} B+\frac{D_{0}^{I V}}{\tanh \lambda_{j} B}\right), n=0,1, \ldots, \infty .
\end{aligned}
$$

There are totally eight groups of unknown coefficients, $C_{q, n}^{I}, C_{j}^{I I}, D_{j}^{I I}, C_{q, n}^{I I}, D_{q, n}^{I I I}, C_{j}^{I V}, D_{j}^{I V}$ and $C_{q, n}^{V}$, which can be determined from a solvable set of linear equations (36), (39), (40), (41), (42), (43), (44), (45) and (46).

\subsection{Expressions of wave exciting forces and wave elevations}

Wave exciting forces on the twin bodies can be obtained by integrating the linear hydrodynamic pressure over the wetted surface of the bodies. Based on the Bernoulli equation, the hydrodynamic pressure in the upper-layer fluid can be evaluated by

$$
p=-\rho_{1} \frac{\partial \Phi^{(1)}}{\partial t}=\rho_{1} i \omega \varphi^{(1)} .
$$

The total horizontal wave exciting force acting on the bodies can be computed by

$$
\begin{aligned}
F_{x} & =\underbrace{\left.\int_{-d}^{0} \rho_{1} i \omega \varphi_{I}^{(1)} d z\right|_{x=-W-B}-\left.\int_{-d}^{0} \rho_{1} i \omega \varphi_{I I I}^{(1)} d z\right|_{x=-(W-B)}}_{F_{x a}} \\
& +\underbrace{\left.\int_{-d}^{0} \rho_{1} i \omega \varphi_{I I I}^{(1)} d z\right|_{x=W-B}-\left.\int_{-d}^{0} \rho_{1} i \omega \varphi_{V}^{(1)} d z\right|_{x=W+B}}_{F_{x b}},
\end{aligned}
$$

where $F_{x a}$ and $F_{x b}$ denote the horizontal wave exciting forces acting on Body a and Body b, respectively.

The total vertical wave exciting force is computed by

$$
F_{z}=\underbrace{\left.\int_{-(W+B)}^{-(W-B)} \rho_{1} i \omega \varphi_{I I}^{(1)} d x\right|_{z=-d}}_{F_{z a}}+\underbrace{\left.\int_{W-B}^{W+B} \rho_{1} i \omega \varphi_{I V}^{(1)} d x\right|_{z=-d}}_{F_{z b}},
$$

where $F_{z a}$ and $F_{z b}$ denote the vertical wave exciting forces acting on Body a and Body b, respectively.

The surface wave elevation can be computed by

$$
\left.\zeta^{S}\right|_{z=0}=-\left.\frac{1}{g} \frac{\partial \Phi^{(1)}}{\partial t}\right|_{z=0}=\left.\frac{i \omega}{g} \varphi^{(1)}\right|_{z=0} .
$$

The internal wave elevation can be evaluated by

$$
\begin{aligned}
\left.\zeta^{I}\right|_{z=-h_{1}} & =-\left.\frac{1}{g} \frac{\frac{\partial \Phi^{(2)}}{\partial t}-\gamma \frac{\partial \Phi^{(1)}}{\partial t}}{1-\gamma}\right|_{z=-h_{1}} \\
& =\left.\frac{i \omega}{g} \frac{\varphi^{(2)}-\gamma \varphi^{(1)}}{1-\gamma}\right|_{z=-h_{1}} .
\end{aligned}
$$


$(a)$

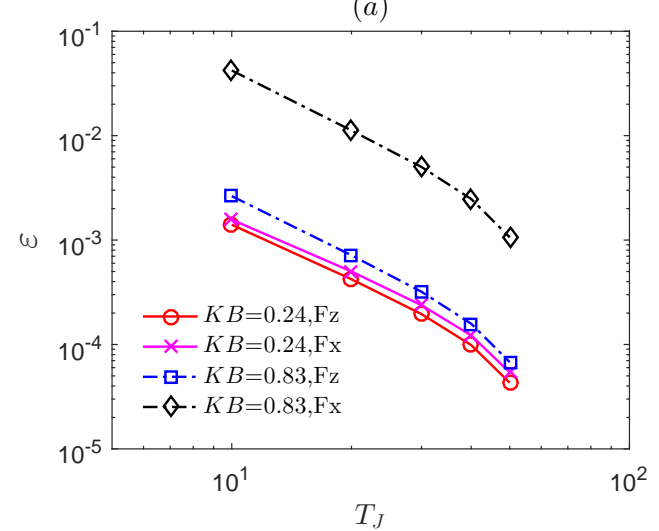

(b)

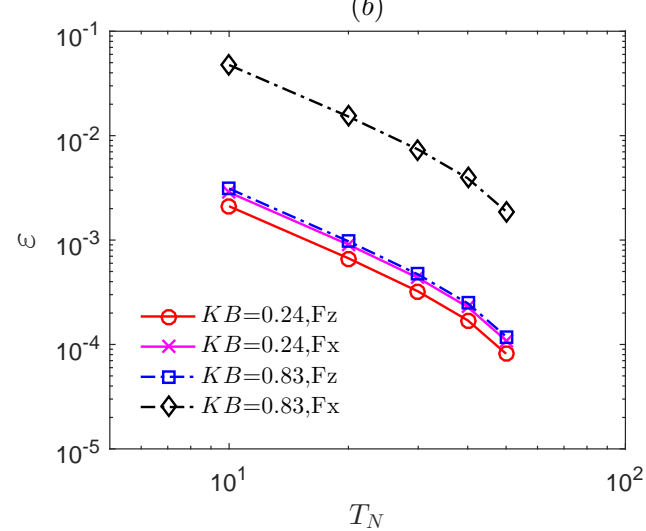

Figure 2: Convergence tests for total horizontal and vertical wave exciting forces. (a): variation of error with respect to truncation order $T_{J} ;(b)$ : variation of error with respect to truncation order $T_{N} \cdot p=1, \gamma=0.9, h_{1} / h_{2}=1, W / B=3, B / d=1, d / h_{1}=0.5$.

\section{Results and discussion}

The wave exciting forces $\left(F_{x}, F_{z}\right)$ and wave elevations $\left(\zeta^{S}, \zeta^{I}\right)$ are computed. $\left|F_{x}\right|,\left|F_{z}\right|,\left|\zeta^{S}\right|,\left|\zeta^{I}\right|$ are the moduli of the wave forces and elevations.

\subsection{Convergence Tests}

The infinite series in the eight sets of linear equations, Eqns. (36), (39), (40), (41), (42), (43), (44), (45) and (46), should be truncated. $T_{N}$ represents the truncation order for $k_{n}^{(p)}$ or $k_{n}^{(q)}$. $T_{J}$ represents the truncation order for $\lambda_{j}$. Convergence tests are presented in Fig. 2 for a typical moonpool configuration. In the convergence tests, one of the truncation orders is varied while the other is fixed. Two wave frequencies are selected for the convergence check in surface wave mode. $K B=0.25$ corresponds to the piston mode resonance frequency and $K B=0.83$ is around the first asymmetric sloshing mode resonance frequency. The relative error $\epsilon$ is defined as $\left|F_{T_{N}}\left(F_{T_{J}}\right)-F_{\max }\right| / F_{\max }$, where $F$ represents the total horizontal or vertical wave exciting force on the twin bodies, and $F_{\max }$ is the corresponding solution with the truncation order $T_{N}$ or $T_{J}=60$. As shown in the log-log plot, the error decreases with the increasing truncation orders, which confirms excellent truncation characteristics of the infinite series. For truncation orders $T_{N}$ and $T_{J}$ being 30 , the relative errors are all less than $0.7 \%$ and the accuracy is acceptable. By balancing the accuracy with the computing time, both truncation orders are set to 30 for the following computations.

\subsection{Validations}

Validation of the present model is carried out by comparing the obtained wave exciting forces and surface wave elevation of a limiting case with the results for a single-layer fluid case. The selected parameters for the limiting case are $p=1, W=0.275, B=0.25, d=0.252, h_{1}=0.5, h_{2}=0.05$ and $\gamma=0.05$, so that it can be compared with the numerical solutions of Ning et al. (2015a,b) for a single-layer fluid. As shown in Fig. 3, the piston mode resonance can be identified at $K h=1.56$. As illustrated in Figs. 3(a) to $(e)$, the predicted piston mode resonance frequency agrees well with that by Ning et al. (2015a,b). The present wave exciting forces and surface wave elevation in resonance region are a little bit larger, which is due to the fact that the present model 
(a)

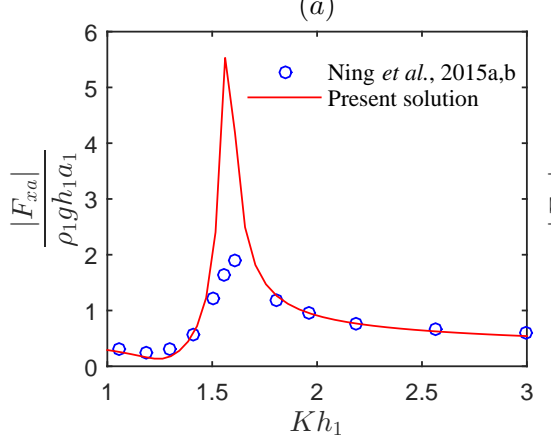

(b)

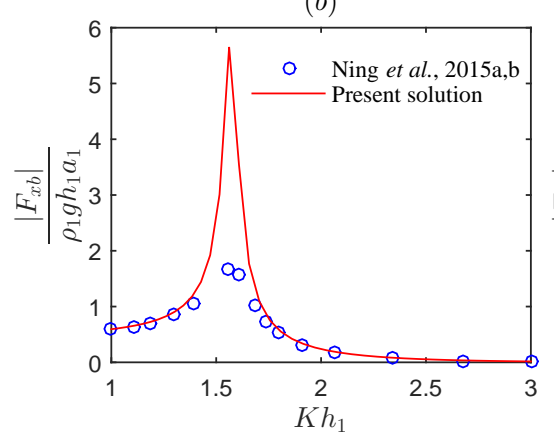

(c)

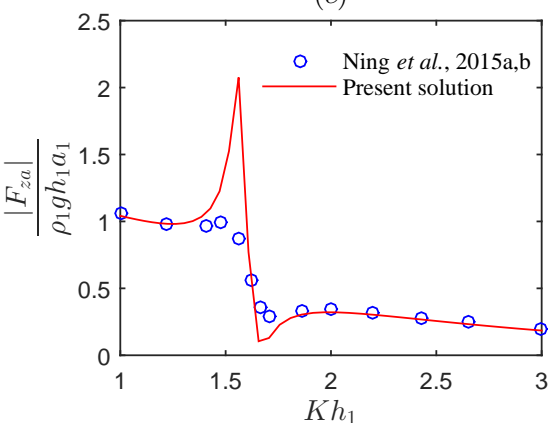

(d)

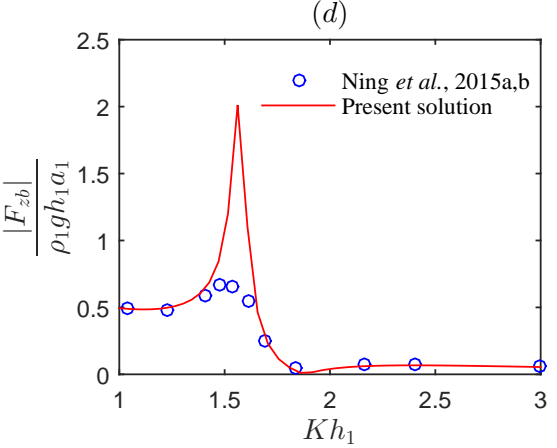

(e)

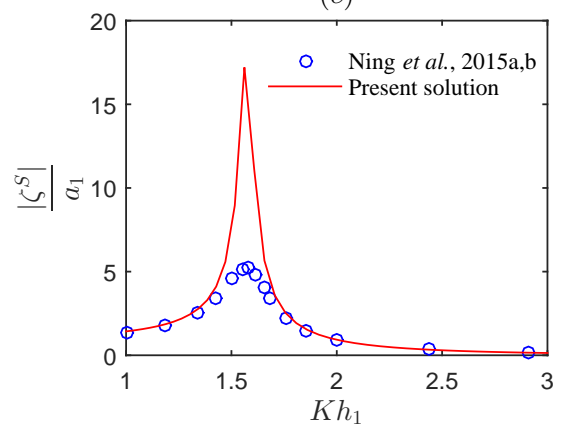

(f)

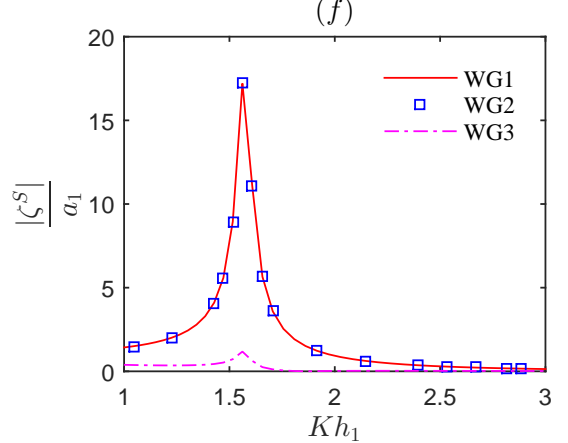

Figure 3: Comparison of wave exciting forces and surface wave elevations for a moonpool with the solutions by Ning et al. (2015a,b). $p=1, \gamma=0.05, W=0.275, B=0.25, d=0.252, h_{1}=0.5, h_{2}=0.05$. (a): horizontal force on Body a; $(b)$ : horizontal force on Body b; $(c)$ : vertical force on Body $\mathrm{a} ;(d)$ : vertical force on Body $\mathrm{b} ;(e)$ : surface wave elevation at the WG1 $(x=0)$; $(f)$ : surface wave elevation at three different numerical wave wave gauges (WG).

can not capture the vortex shedding at the keel of the twin boxes, while an artificial damping is adopted on the free surface boundary condition in Ning's model. In addition, as shown in Figs. 3(a) to $(e)$, the surface wave elevations, horizontal and vertical wave exciting forces by the two methods agree well with each other in non-resonance region.

\subsection{Wave exciting forces and wave elevations}

A typical moonpool with $W / B=3.0$ is selected for computations and analyses. The exciting forces and wave elevations in surface wave mode are illustrated in Fig. 4. Of particular interest in this figure are the three resonances around $K B=0.24,0.83$ and 1.57 . To identify their corresponding resonance modes, the wave elevations inside the moonpool in the neighborhood of these points are plotted in Fig. 5.

As can be seen in Figs. 5(a) and $(b)$, the surface wave elevation inside the moonpool are relatively flat. Hence, the resonance around $K B=0.24$ can be identified as a piston mode resonance. As shown in Figs. $5(c)$ and $(d)$, the surface wave elevation is nil at the center of moonpool, while the maxima are reached at $x= \pm(W-B)$. In addition, the wave profile inside the moonpool is in half sinusoidal form so that antisymmetric sloshing mode can be identified around $K B=0.83$. In contrast, for the third higher-order resonance, as shown in Figs. 5(e) and $(f)$, the surface wave elevation reaches the maxima at both the center of the moonpool and $x= \pm(W-B)$. As shown, the modal shape is approximately in full sinusoidal form so that the resonance around $K B=1.57$ is of symmetric sloshing mode. 
The wavelengths $\lambda_{l}$ for these sloshing mode resonances should meet

$$
\frac{l}{2} \lambda_{l}=2(W-B), \quad l=1,2, \ldots, \infty
$$

Based on the dispersion relation (3), an estimation formula of sloshing mode resonance frequencies in surface wave mode $(p=1)$ can be derived

$$
K B=\frac{k B}{2\left(1+\gamma t_{1} t_{2}\right)}\left\{t_{1}+t_{2}+\sqrt{\left(t_{1}+t_{2}\right)^{2}-4 \varepsilon t_{1} t_{2}\left(1+\gamma t_{1} t_{2}\right)}\right\},
$$

where $k=2 \pi / \lambda_{l},(l=1,2, \ldots, \infty), t_{1}=\tanh k h_{1}, t_{2}=\tanh k h_{2}$. By using Eqn. (53), the predicted resonance frequencies for the first two sloshing modes are $K B=0.78$ and 1.57 , respectively. The sloshing mode resonance frequencies in high-frequency region can be estimated by using Eqn. (53), though resonance frequency of the first sloshing mode is a little bit underestimated. The difference for the first sloshing mode resonance can be attributed to the fact that the wavelengths at low-frequency are much larger than the draft of the twin bodies, such that the side walls of the twin bodies can not be considered as deep vertical walls. As shown in Fig. 4(a), the bandwidth of sloshing mode resonances decreases with the increasing wave frequency.

As shown in Fig. 4(a), around the piston mode resonance frequency, the non-dimensional free surface elevation inside the moonpool and at the weather side of Body a (WG5) is around 1.8, while the free surface elevation at the leeward side of Body b (WG3) is about 1.0. In Fig. 4(b), both antisymmetric sloshing mode and symmetric sloshing mode resonances can be observed in internal wave elevation. In addition, distinct from the surface wave elevation, the internal wave elevation reaches its maximum in low-frequency region, i.e. $K B<0.1$. Interestingly, in low-frequency region, the internal wave elevation can be larger than the surface wave elevation in surface wave mode.

As shown in Fig. 4(c), the total horizontal force is nonzero around piston mode resonance frequency, and smaller than the horizontal force components $\left(F_{x a}\right.$ and $\left.F_{x b}\right)$. As shown in Fig. $4(d)$, the total vertical force around piston mode resonance is about the sum of the vertical force components $\left(F_{z a}\right.$ and $\left.F_{z b}\right)$ on the two bodies. As the wave frequency exceeds the piston mode resonance frequency, the vertical forces exhibit a sharp drop, and the total vertical force reduces to almost zero.

Fig. 6 shows the wave exciting forces and wave elevations in internal wave mode with $\gamma=0.9$. Different from the case in surface wave mode, no obvious resonances can be observed. This is because when $\gamma$ approaches to $1.0, k_{0}^{(2)}$ is much larger than $k_{0}^{(1)}$. Consequently, the wavelength of internal wave is too small, compared to the moonpool width, to trigger resonances. In addition, the wave exciting forces show more humps in low-frequency area. As the wave frequency increases, both wave elevations and wave exciting forces attenuate very quickly. For this moonpool configuration, as $K B$ exceeds 0.2 , the surface wave elevation, horizontal forces and vertical forces diminish, whereas the internal wave elevation approaches to 1.0. This indicates that the incident internal wave does not have significant impact on the wave exciting forces and wave motions inside moonpool domian at high frequencies. As illustrated in Fig. 4(b) and Fig. 6(b), the internal wave elevation exhibits a few peaks and valleys in both wave modes. 
$(a)$

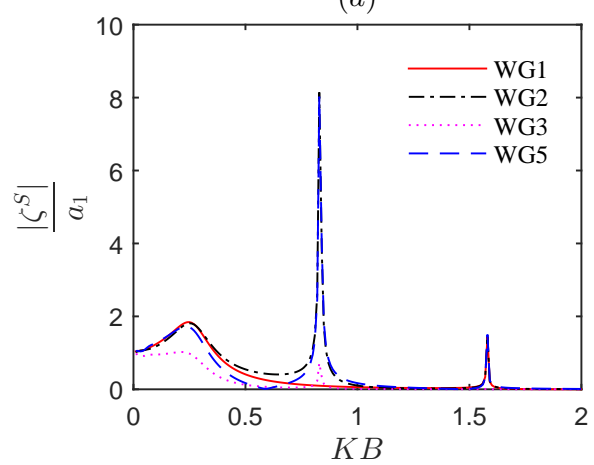

$(c)$

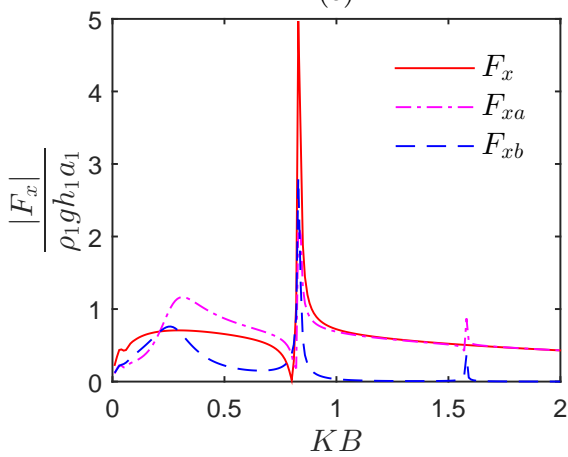

(b)

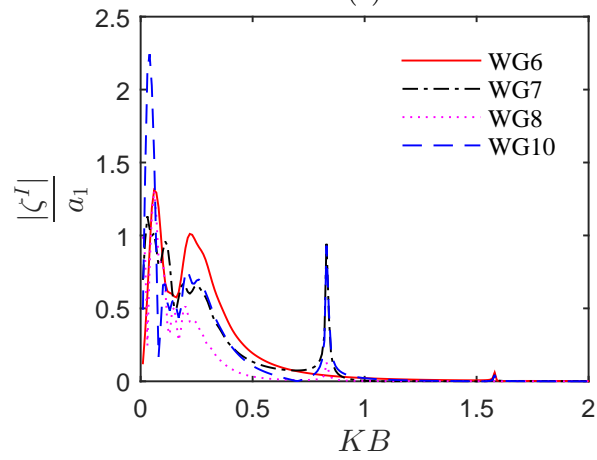

$(d)$

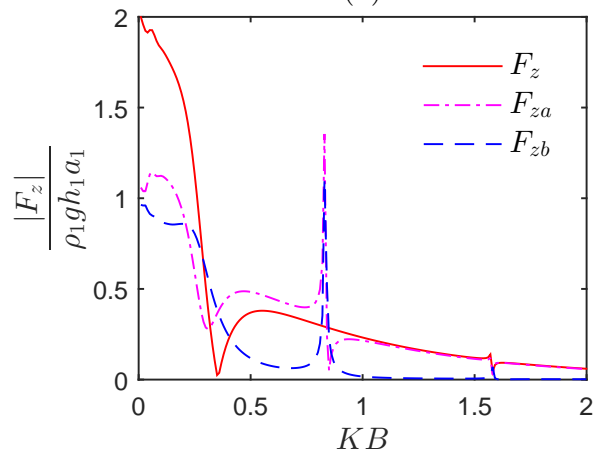

Figure 4: Wave elevations and wave exciting forces in surface wave mode. $\gamma=0.9, h_{1} / h_{2}=1.0, W / B=3.0, B / h_{1}=0.5$, $d / h_{1}=0.5$. (a): surface wave elevation; $(b)$ : internal wave elevation; $(c)$ : horizontal force; $(d)$ : vertical force.

(a)

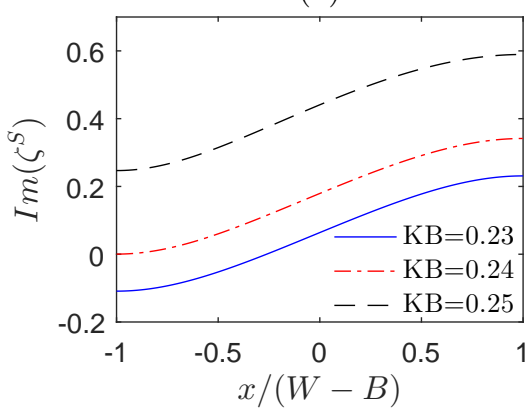

(b)

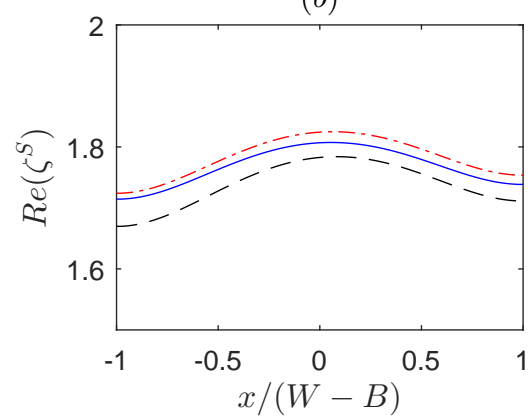

$(c)$

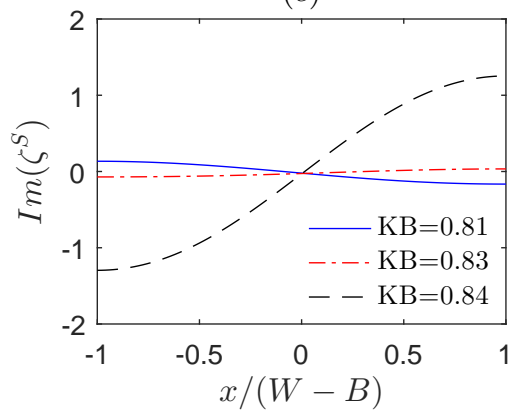

$(d)$

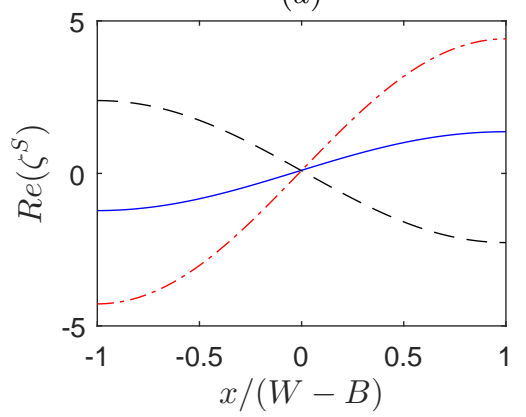

$(e)$



$(f)$

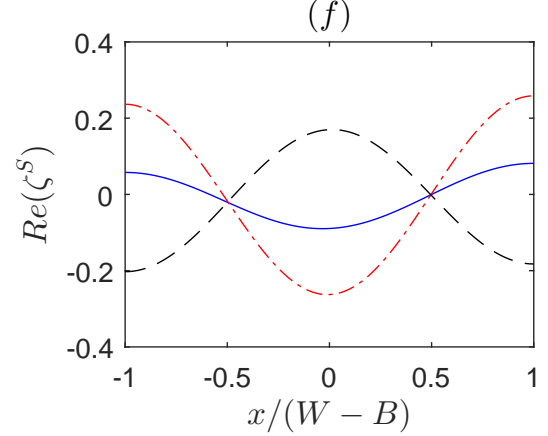

Figure 5: Modal shapes of piston and sloshing modes in surface wave mode $(p=1) \cdot \gamma=0.9, h_{1} / h_{2}=1.0, W / B=3.0, B / h_{1}=0.5$, $d / h_{1}=0.5$. (a): imaginary component for piston mode; $(b)$ : real component for piston mode; $(c)$ : imaginary component for antisymmetric sloshing mode; $(d)$ : real component for antisymmetric sloshing mode; $(e)$ : imaginary component for symmetric sloshing mode; $(f)$ : real component for symmetric sloshing mode. 
(a)



$(c)$

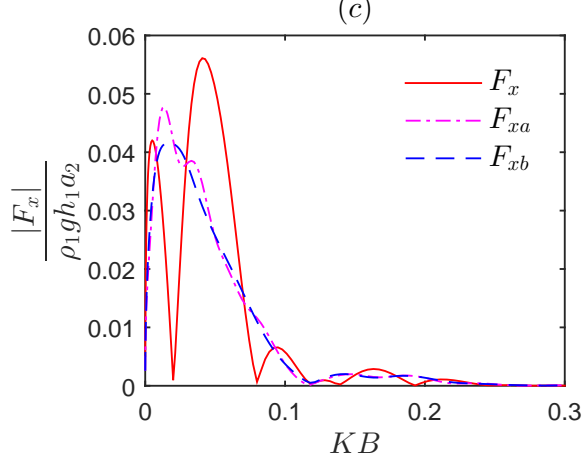

(b)

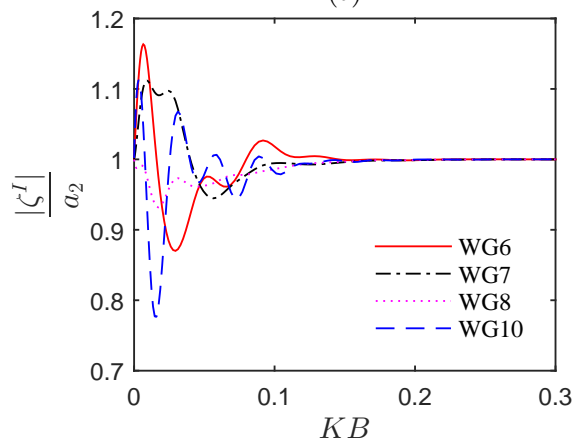

$(d)$

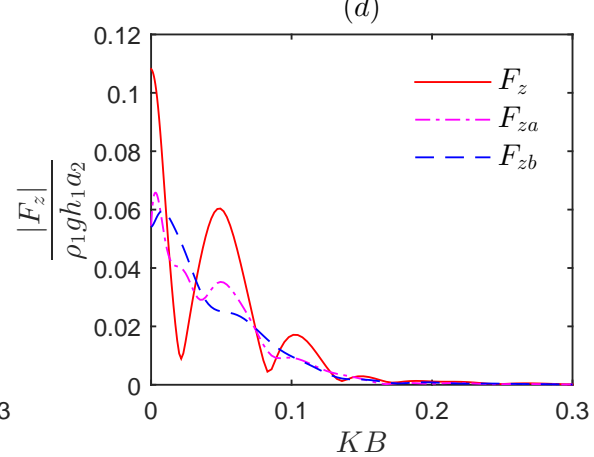

Figure 6: Wave elevations and wave exciting forces in internal wave mode. $\gamma=0.9, h_{1} / h_{2}=1.0, W / B=3.0, B / h_{1}=0.5$, $d / h_{1}=0.5$. (a): surface wave elevation; $(b)$ : internal wave elevation; $(c)$ : horizontal force; $(d)$ : vertical force.

\subsection{Parametric studies}

\subsubsection{Effects of density ratio}

To study the effects of the density ratio, the solutions for $\gamma=0.3,0.7,0.9$ and 1.0 are analyzed. When $\gamma=1.0$, the two-layer fluid becomes a single-layer fluid of depth $h=h_{1}+h_{2}$. The predictions for $\gamma=1.0$ are computed by the code for solving the diffraction problem of moonpool in single-layer fluid. The details can be referred to Yeung and Seah (2007). The draft of twin bodies is set to $d / h_{1}=0.9$, so that the bottoms of the bodies are close to the interfacial surface and the effects of internal waves on wave motions inside the moonpool are examined. The wave exciting forces and wave elevations with different density ratios $\gamma$ in surface wave mode and internal wave mode are illustrated in Figs. 7 and 8, respectively. As can be seen from the plots, the density ratio has little effects on the sloshing mode resonance frequencies in both surface and internal wave modes.

As shown in Fig. $7(e)$, in surface wave mode, as the density ratio decreases gradually from 1.0 to 0.7 , the piston mode resonance frequency increases slightly while the surface wave elevations around this resonance region decrease a little bit. This means that the decreasing density ratio can somehow suppress the resonant responses. However, in high-frequency region, the stratification has little effects on the sloshing mode resonance frequencies.

As shown in Fig. 8, the decrease of density ratio can lead to remarkable increase in the magnitudes of surface wave elevation and wave exciting forces. The reason is that, as density ratio decreases, the density of the upper-layer fluid tends to be small so that the energy can be easily transferred from internal wave mode to surface wave mode (Linton and McIver, 1995). As discussed previously, for $\gamma=0.9$, no obvious sloshing 
(a)

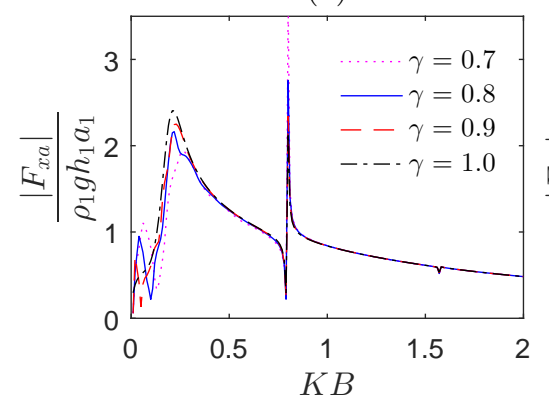

(b)

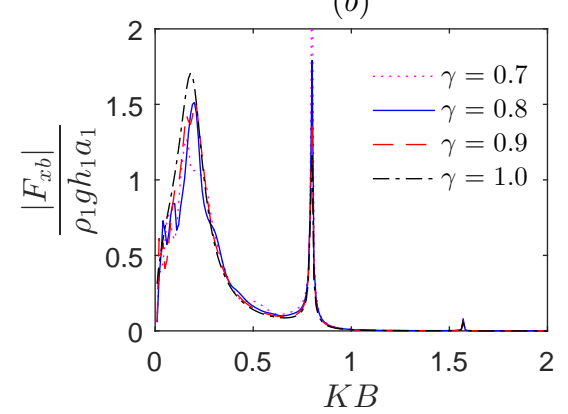

(c)



(d)

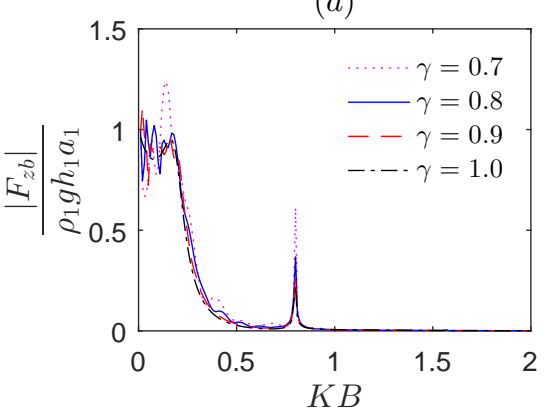

(e)

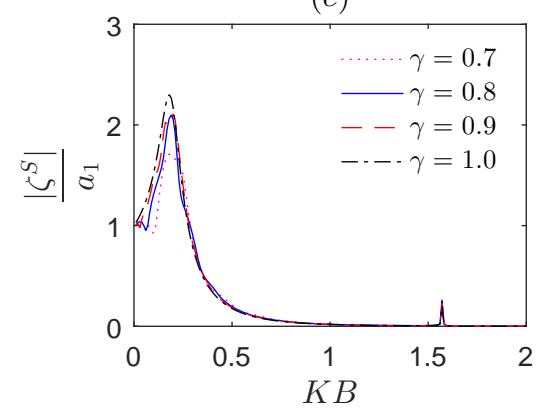

(f)

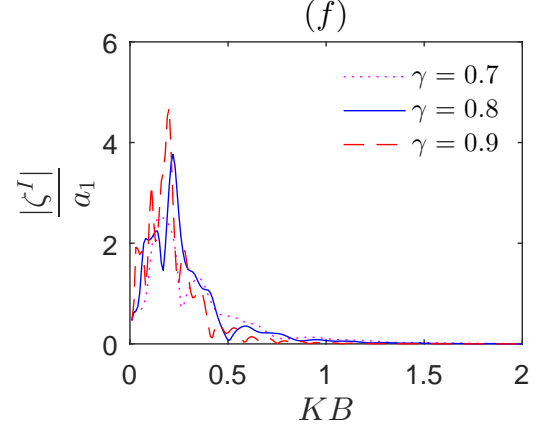

Figure 7: Wave exciting forces and wave elevations for moonpools with different $\gamma$ in surface wave mode. $h_{1} / h_{2}=1.0, W / B=3.0$, $B / h_{1}=0.5, d / h_{1}=0.9$. $(a)$ : horizontal force on Body a; $(b)$ : horizontal force on Body b; $(c)$ : vertical force on Body a; $(d)$ : vertical force on Body $\mathrm{b} ;(e)$ : surface wave elevation at WG1; $(f)$ : internal wave elevation at WG6.

mode resonances can be observed in internal wave mode, and the effects of the internal waves are concentrated within low-frequency region. However, when the density ratio decreases, the sloshing mode resonances become evident and the resonant responses are amplified. The comparison of the Figs. 7 and 8 suggests that the sloshing resonance frequencies in internal wave mode are consistent with those in surface wave mode. As shown in Fig. $8(f)$, when wave frequency exceeds a critical value for each $\gamma$, the internal wave elevation tends to be 1.0 in internal wave mode. Furthermore, in internal wave mode, the frequency region where the internal wave elevation can be affected becomes broader as the density ratio decreases.

\subsubsection{Effects of depth ratio}

To study the effects of the depth ratio, the solutions for $h_{2} / h_{1}=0,0.5,1.0$ and 2.0 are analyzed. When $h_{2} / h_{1}=0$, the two-layer fluid becomes a single-layer fluid of depth $h=h_{1}$. The predictions for $h_{2} / h_{1}=0$ are computed by the code for solving the diffraction problem of moonpool in single-layer fluid. The wave exciting forces and wave elevations for moonpool with respect to depth ratio $h_{2} / h_{1}$ in surface and internal wave modes are presented in Figs. 9 and 10, respectively. In these cases, the depth of upper-layer fluid is kept the same.

As shown in Fig. 9(e), the piston mode resonance frequency increases with the rising of depth ratio in surface wave mode, while there is no obvious variation in sloshing mode resonance frequencies. The reason is that, based on dispersion relation $(3), k_{0}^{(1)}$ decreases as the depth ratio increases in low-frequency region, though it is rarely affected in high-frequency region. Since $k_{0}^{(1)}$ is significant for moonpool resonance, the fluid inside the moonpool with larger depth ratio reaches the natural frequency of moonpool at smaller $\omega$, triggering the piston resonance. The sloshing mode resonances which occur at relatively higher frequencies are rarely affected. 
(a)

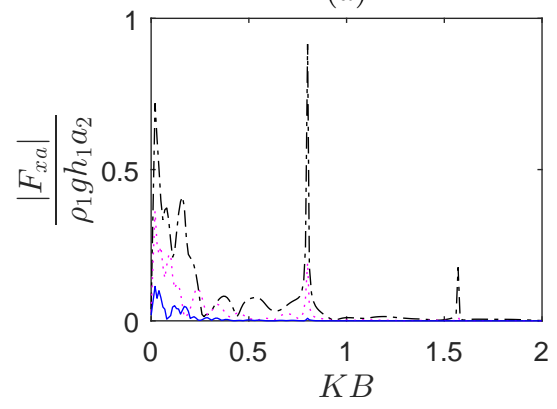

$(b)$

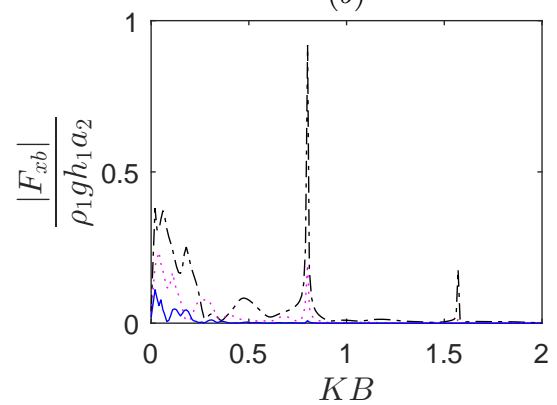

$(c)$



$(d)$

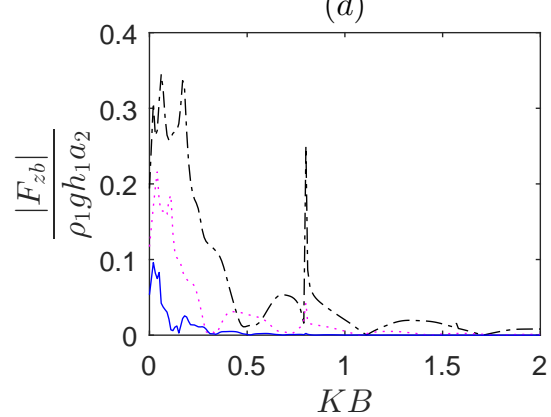

$(e)$

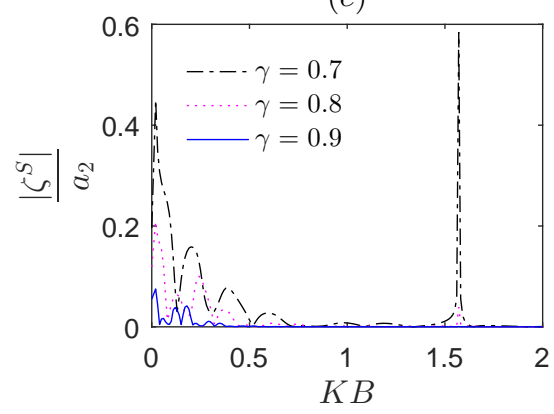

$(f)$

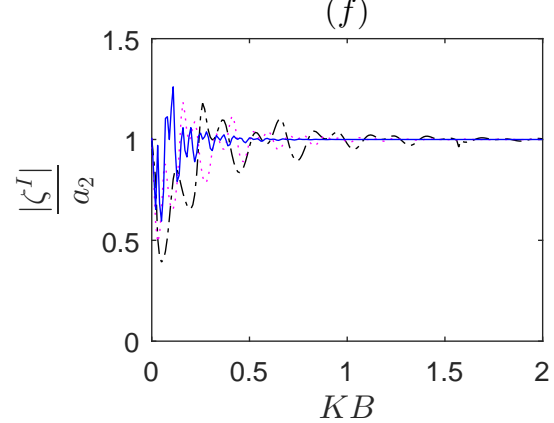

Figure 8: Wave exciting forces and wave elevations for moonpools with different $\gamma$ in internal wave mode. $h_{1} / h_{2}=1.0, W / B=3.0$, $B / h_{1}=0.5, d / h_{1}=0.9$. $(a)$ : horizontal force on Body a; $(b)$ : horizontal force on Body b; $(c)$ : vertical force on Body a; $(d)$ : vertical force on Body $\mathrm{b} ;(e)$ : surface wave elevation at WG1; $(f)$ : internal wave elevation at WG6.

In addition, as shown in Fig. 9(e), around the piston mode resonance region, the depth ratio has little effects on surface wave amplitude. However, as shown in Fig. 9(f), the resonant internal wave amplitude becomes larger when the depth ratio increases. This is due to the fact that the amplitude ratio $\alpha\left(k_{0}^{(1)}\right)$ increases as the piston mode resonance frequency $\omega$ increases while $k_{0}^{(1)}$ is kept the same (see Eqn. (20)).

Fig. 10 shows that, in general, for internal wave mode, the wave exciting forces and surface wave elevations increase significantly with increasing $h_{2} / h_{1}$ at low-frequency region. This is due to the fact that, in low-frequency region, the incident internal waves have more kinetic energy in deeper fluid depth, so that more energy can be transferred from internal wave mode to the surface wave mode. Hence, the wave exciting forces and wave elevations increase as the depth of lower-layer increases.

\subsubsection{Effects of moonpool width}

The variations of the wave exciting forces and wave elevations with respect to $(W-B)$ are illustrated in Figs. 11 and 12 for surface and internal wave modes, respectively. Fig. 11 shows that, in surface wave mode, the moonpool width has significant effects on both piston and sloshing mode resonances. As can be observed in Figs. 11 $(a)$ and $(b)$, around the piston mode resonance frequency, the horizontal wave exciting forces decreases with the increasing moonpool width. However, as shown in Figs. 11(c) and $(d)$, the maximum vertical forces on each body remain unchanged. In addition, as shown in the plots of wave exciting forces, the sloshing mode resonances become more broad-banded as moonpool width increases.

Fig. 11 shows that as the moonpool width increases, both piston and sloshing mode resonance frequencies are reduced. This can be attributed to the fact that the corresponding wavelength for each sloshing mode 
(a)

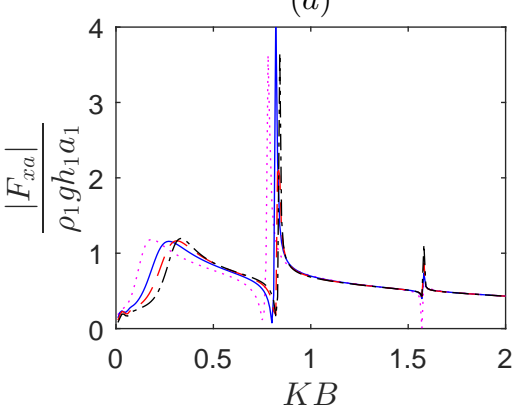

(b)



(c)

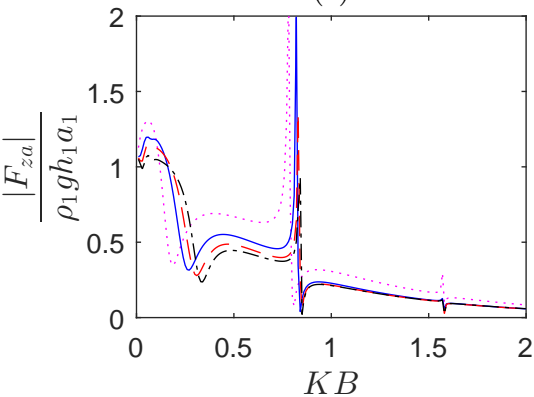

(d)

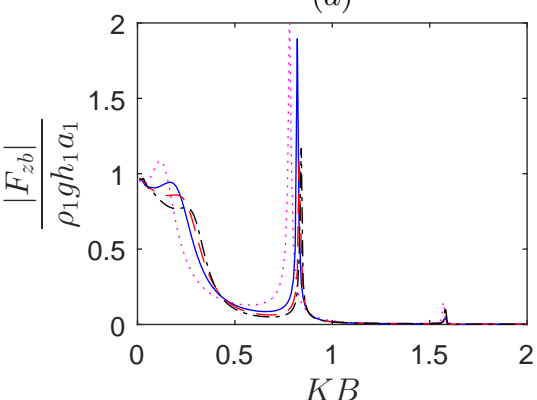

(e)

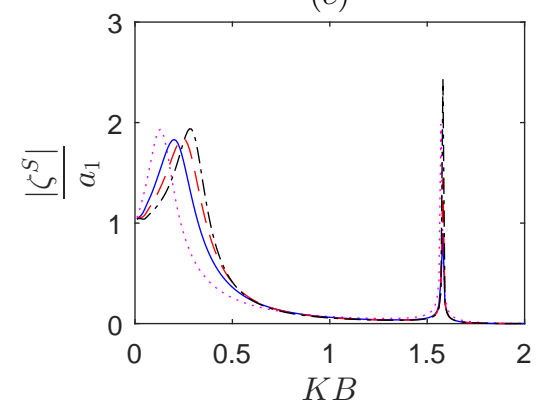

(f)

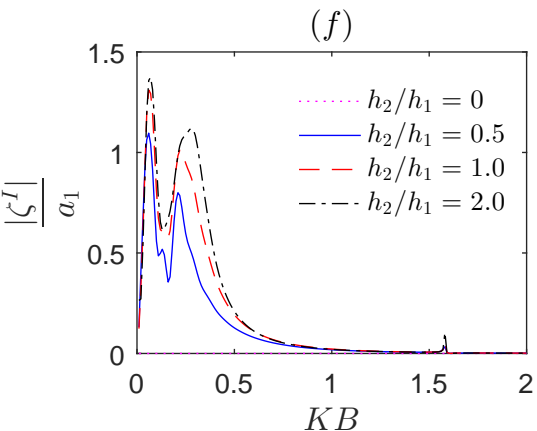

Figure 9: Wave exciting forces and wave elevations for moonpools with different $h_{2} / h_{1}$ in surface wave mode. $\gamma=0.9, W / B=3.0$, $B / h_{1}=0.5, d / h_{1}=0.5$. $(a)$ : horizontal force on Body a; $(b)$ : horizontal force on Body b; $(c)$ : vertical force on Body a; $(d)$ : vertical force on Body $\mathrm{b} ;(e)$ : surface wave elevation at WG1; $(f)$ : internal wave elevation at WG6.

(a)

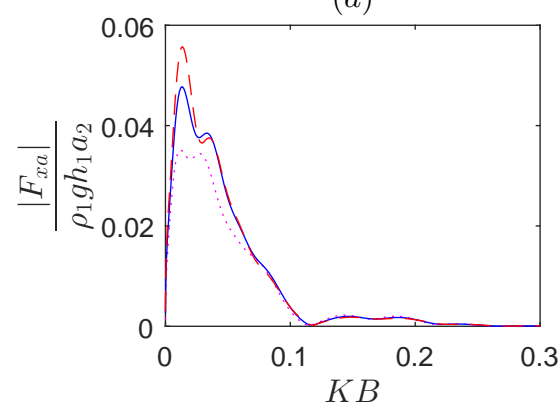

(b)

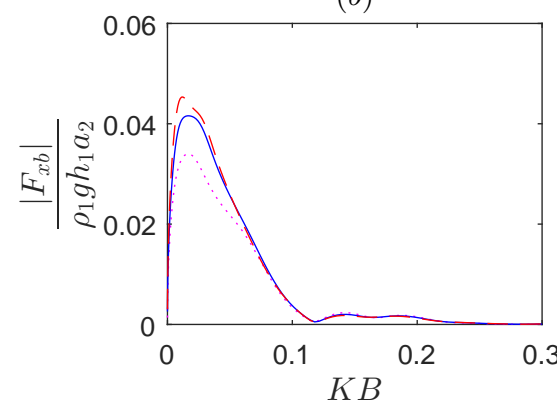

(c)



$(d)$

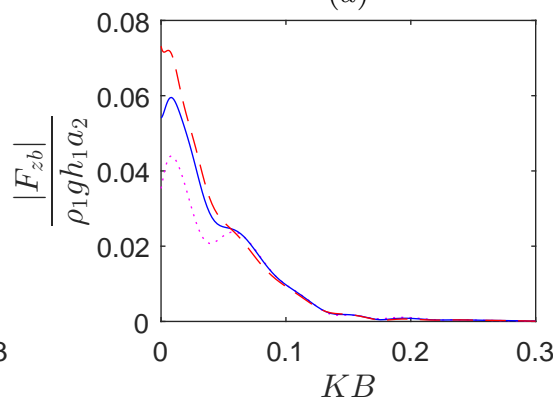

$(e)$

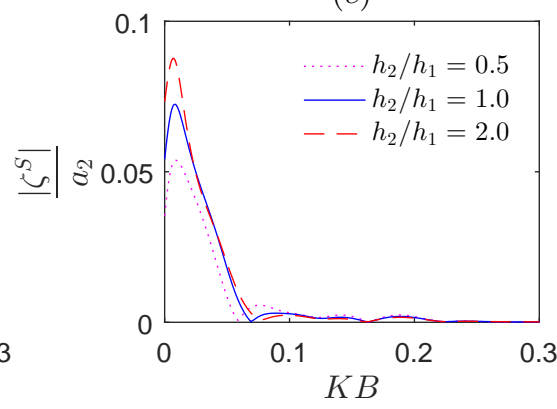

$(f)$

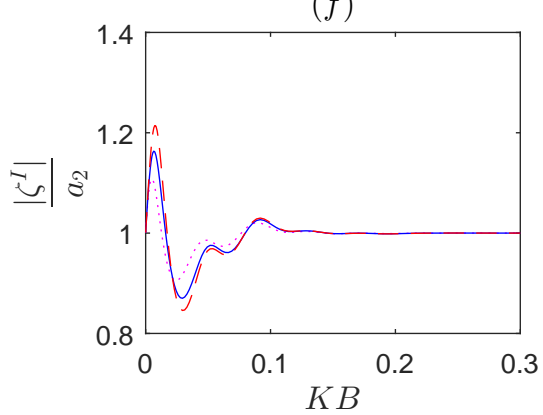

Figure 10: Wave exciting forces and wave elevations for moonpools with different $h_{2} / h_{1}$ in internal wave mode. $\gamma=0.9, W / B=3.0$, $B / h_{1}=0.5, d / h_{1}=0.5$. (a): horizontal force on Body a; $(b)$ : horizontal force on Body b; $(c)$ : vertical force on Body a; $(d)$ : vertical force on Body $\mathrm{b} ;(e)$ : surface wave elevation at WG1; $(f)$ : internal wave elevation at WG6. 
(a)

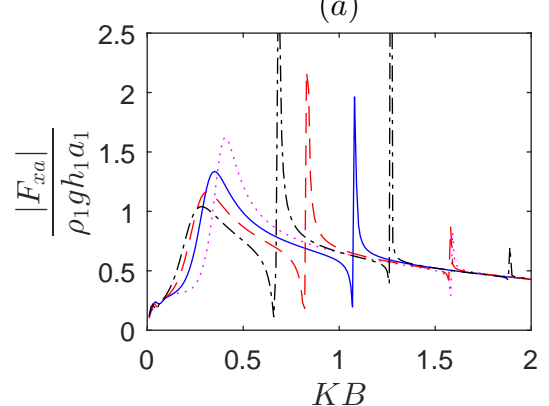

(b)

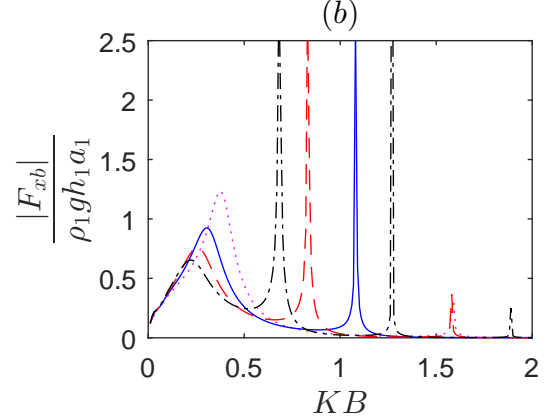

(c)



$(d)$

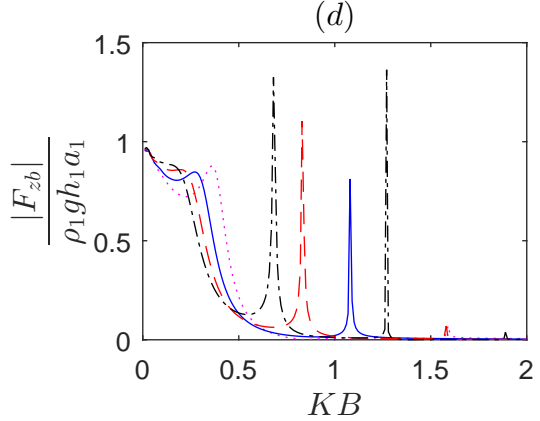

$(e)$

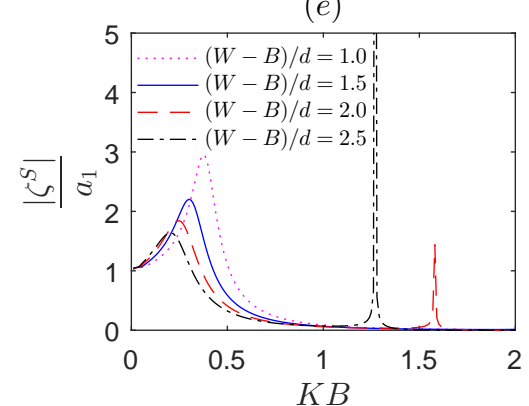

$(f)$



Figure 11: Wave exciting forces and wave elevations for moonpools with different $(W-B) / d$ in surface wave mode. $\gamma=0.9$, $h_{1} / h_{2}=1.0, B / h_{1}=0.5, d / h_{1}=0.5$. $(a)$ : horizontal force on Body a; $(b)$ : horizontal force on Body b; $(c)$ : vertical force on Body a; $(d)$ : vertical force on Body b; $(e)$ : surface wave elevation at WG1; $(f)$ : internal wave elevation at WG6.

resonance increases as the moonpool width increases. For piston mode resonance, as moonpool width increases, the mass of fluid inside moonpool domain increases so that the natural frequency of moonpool decreases. The variation of moonpool resonance frequencies with respect to moonpool width is consistent with that observed by Zhang et al. (2019) (Fig. 14 therein) for the case of single-layer fluid. As shown in Figs. 11(e) and (f), the piston mode resonant motions of surface and internal wave elevations are suppressed significantly as moonpool width increases.

Fig. 12 suggests that the effects of moonpool width on the magnitudes of the wave exciting forces and wave elevations are not significant in internal wave mode, but more humps can be observed with the increasing moonpool width.

\subsubsection{Effects of body width}

The wave exciting forces and wave elevations for moonpools with different body widths $B$ in surface and internal wave modes are presented in Figs. 13 and 14, respectively. In these cases, the moonpool width $(W-B)$ is kept unchanged.

In surface wave mode, as shown in Figs. 13(e) and $(f)$, the piston mode resonance frequency (with $K h_{1}$ close to 0.5 ) increases slightly as the body width decreases, while the sloshing mode resonance frequencies are rarely affected due to the unchanged moonpool width. This phenomenon is consistent with that observed by Zhang et al. (2019) (Fig. 16 therein) for the case of single-layer fluid. In addition, as shown in Fig. 13(e), the wave motion around piston mode resonance region are suppressed as the body width decreases. As illustrated in Figs. 13(a) and (b), the horizontal wave exciting force decreases in piston mode resonance region as the body width reduces. As shown in Figs. 13(c) and $(d)$, the vertical wave exciting forces decrease significantly near the 
(a)

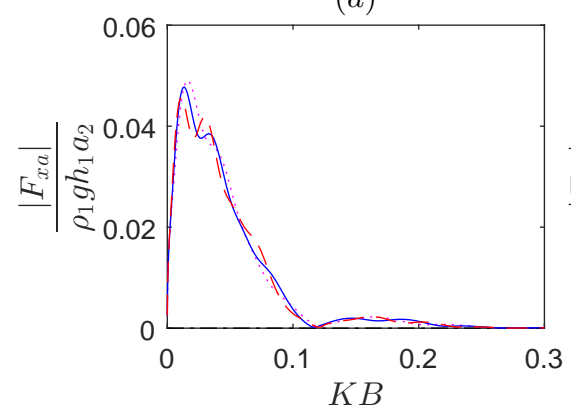

(b)

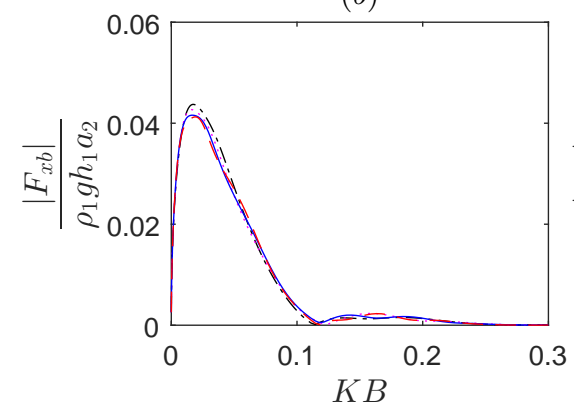

(c)

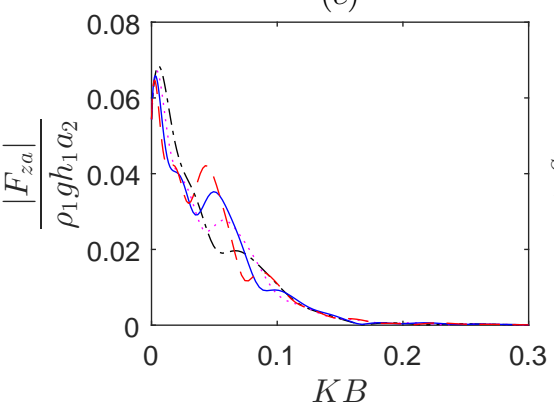

(d)

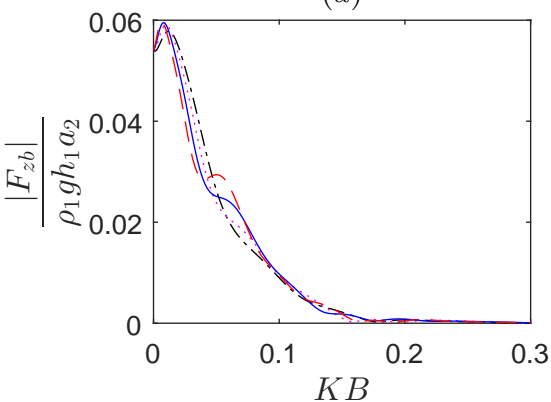

(e)



$(f)$



Figure 12: Wave exciting forces and wave elevations for moonpools with different $(W-B) / d$ in internal wave mode. $\gamma=0.9$, $h_{1} / h_{2}=1.0, B / h_{1}=0.5, d / h_{1}=0.5$. $(a)$ : horizontal force on Body a; $(b)$ : horizontal force on Body b; $(c)$ : vertical force on Body $\mathrm{a} ;(d)$ : vertical force on Body b; $(e)$ : surface wave elevation at WG1; $(f)$ : internal wave elevation at WG6.

piston and sloshing mode resonance frequencies. One reason is that the wetted area (body width) is reduced. Another reason may be that, as the piston mode resonance frequency increases, the fluid velocity attenuates more quickly towards the negative direction of $z$-axis and the hydrodynamic pressure at the bottom of twin bodies decreases.

In internal wave mode, as shown in Fig. 14, more peaks and valleys can be observed in wave exciting forces and wave elevations as the body width increases. Meanwhile their maximum amplitudes in low-frequency region increase.

\subsubsection{Effects of draft}

The wave exciting forces and wave elevations for moonpools with different body drafts in surface and internal wave modes are presented in Figs. 15 and 16, respectively. Figs. $15(a)$ and $(b)$ show that, in surface wave mode, around the piston mode resonance region, the horizontal wave exciting forces increase with the rising of the draft for the sake of larger wetted area. However, as illustrated in Figs. 15(c) and $(d)$, the vertical wave exciting forces decrease with the increasing draft in piston mode resonance region, because the hydrodynamic pressure attenuates along the negative direction of $z$-axis.

As shown in Fig. 15(e), in surface wave mode, both the piston and sloshing mode resonance frequencies reduce as draft increases. However, when the draft exceeds a critical value, the sloshing mode resonances seems to keep the same. This phenomenon is consistent with that observed by Zhang et al. (2019) (Fig. 15 therein) for the case of single-layer fluid. This can be attributed the fact that the fluid trapped in the moonpool increases and the natural frequency of moonpool is reduced. In addition, as the body draft increases, the fluid can be 
(a)

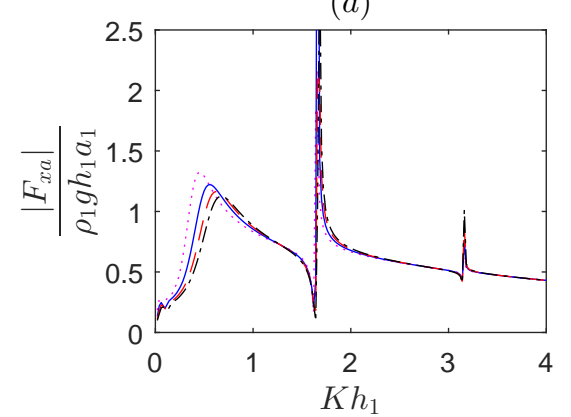

(b)

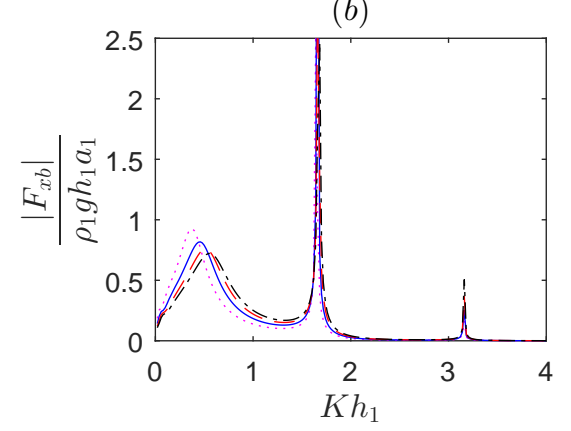

(c)

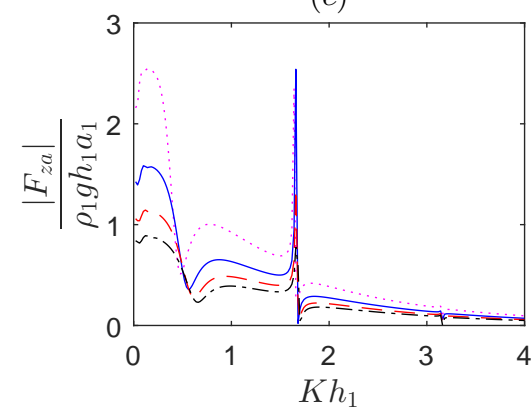

(d)

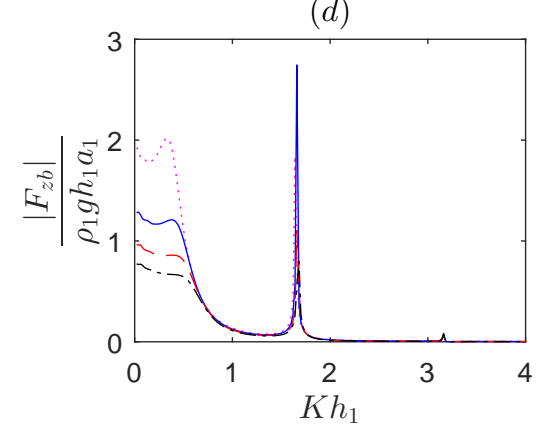

(e)

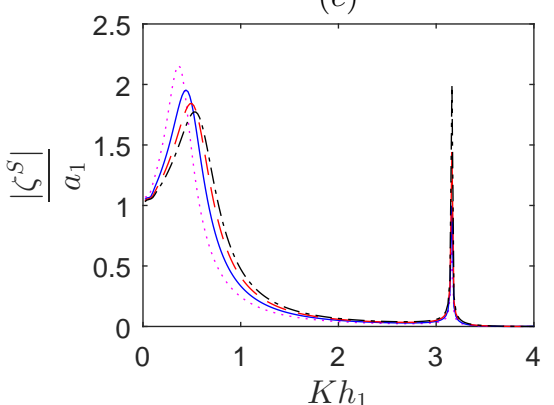

(f)

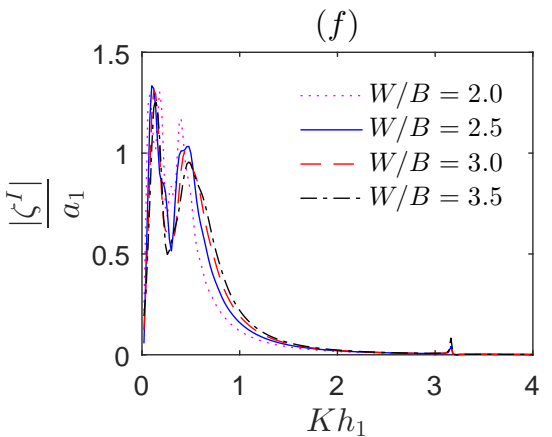

Figure 13: Wave exciting forces and wave elevations for moonpools with different $W / B$ in surface wave mode. $\gamma=0.9, h_{1} / h_{2}=1.0$, $(W-B) / d=2.0, d / h_{1}=0.5$. $(a)$ : horizontal force on Body a; $(b)$ : horizontal force on Body b; $(c)$ : vertical force on Body a; $(d)$ : vertical force on Body b; $(e)$ : surface wave elevation at WG1; $(f)$ : internal wave elevation at WG6.

(a)



(b)

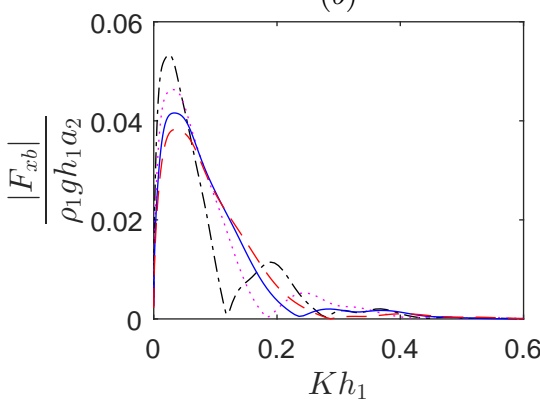

(c)

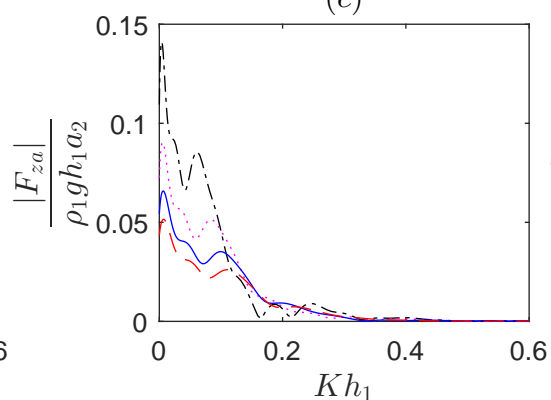

(d)

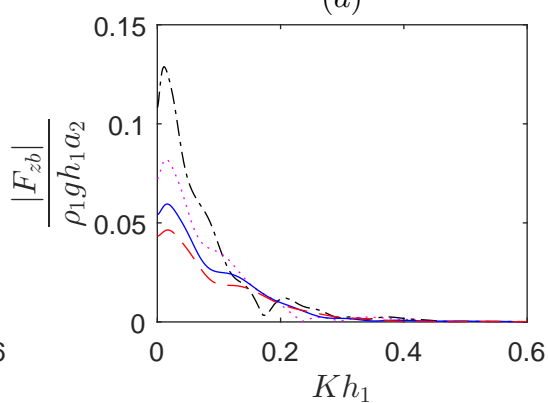

(e)



(f)

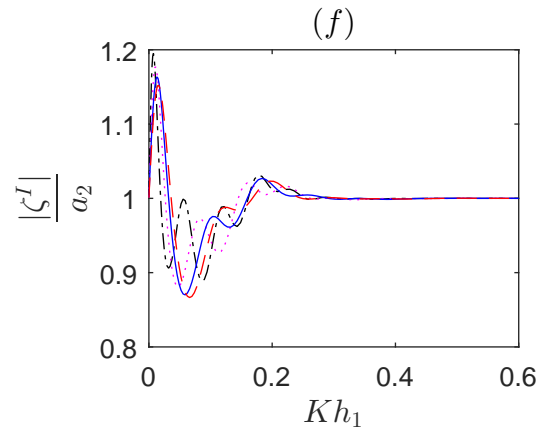

Figure 14: Wave exciting forces and wave elevations for moonpools with different $W / B$ in internal wave mode. $\gamma=0.9, h_{1} / h_{2}=1.0$, $(W-B) / d=2.0, d / h_{1}=0.5$. $(a)$ : horizontal force on Body a; $(b)$ : horizontal force on Body b; $(c)$ : vertical force on Body a; $(d)$ : vertical force on Body $\mathrm{b} ;(e)$ : surface wave elevation at WG1; $(f)$ : internal wave elevation at WG6. 
$(a)$



$(b)$



(c)

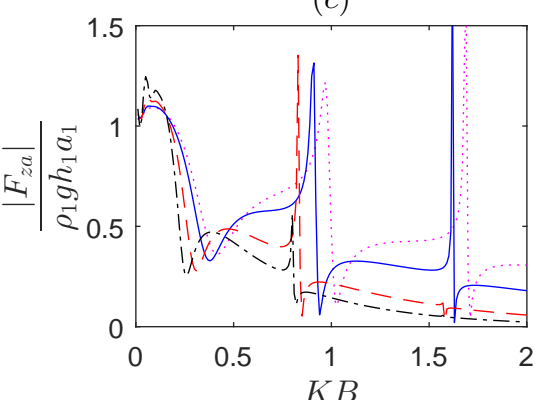

$(d)$

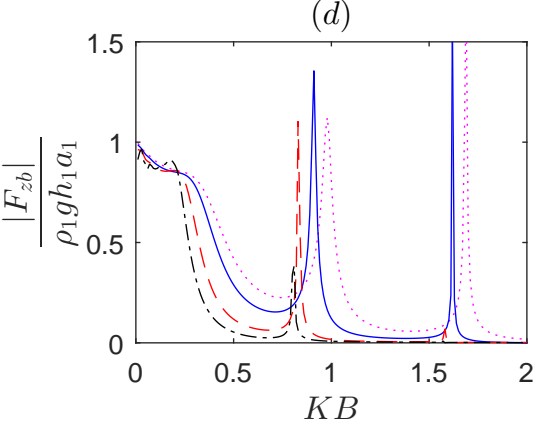

$(e)$

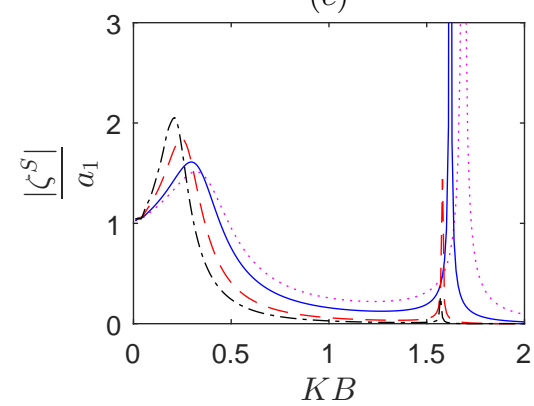

$(f)$

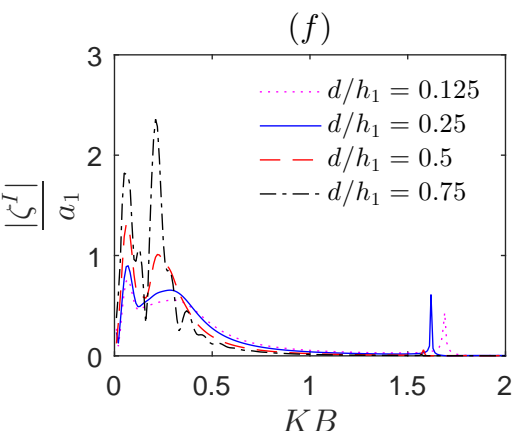

Figure 15: Wave exciting forces and wave elevations for moonpools with different $d / h_{1}$ in surface wave mode. $\gamma=0.9, h_{1} / h_{2}=1.0$, $W / B=3.0, B / h_{1}=0.5$. $(a)$ : horizontal force on Body a; $(b)$ : horizontal force on Body b; $(c)$ : vertical force on Body a; $(d)$ : vertical force on Body $\mathrm{b} ;(e)$ : surface wave elevation at WG1; $(f)$ : internal wave elevation at WG6.

easily trapped inside the moonpool domain. Therefore, as observed in Figs. 15(e) and $(f)$, the wave motions around piston mode resonance region are intensified with the increasing $d / h_{1}$. Moreover, both piston and sloshing mode resonance regions become more narrow-banded as the draft increases.

In internal wave mode, as presented in Fig. 16, both the wave exciting forces and free surface wave elevations are amplified as draft increases. Fig. 16(f) shows that, as the draft increases and the bottoms of the twin bodies approach the interfacial surface, the frequency region where the internal wave elevation can be affected becomes larger.

\section{Conclusions}

In this paper, the wave diffraction problems of two-dimensional moonpools in a two-layer fluid with finite depth have been studied using the method of eigenfunction expansion and domain decomposition. The present model is validated by comparing a limiting case with the single-layer fluid case. Parametric studies are performed to investigate the effects of density ratio, depth ratio, moonpool width, body width and draft. Through extensive computations and analyses, it is found that

1) Wave exciting forces in internal wave mode are much smaller than those in surface wave mode. In addition, in internal wave mode, the wave exciting forces and wave elevations show more peaks and valleys. The effects of internal waves are concentrated within low-frequency region. For twin bodies with deep draft in surface wave mode, the presence of stratification can suppress the horizontal wave exciting forces and surface wave elevations around piston mode resonance region.

2) As the wave frequency increases, the bandwidth of sloshing mode resonance decreases. The sloshing 
(a)

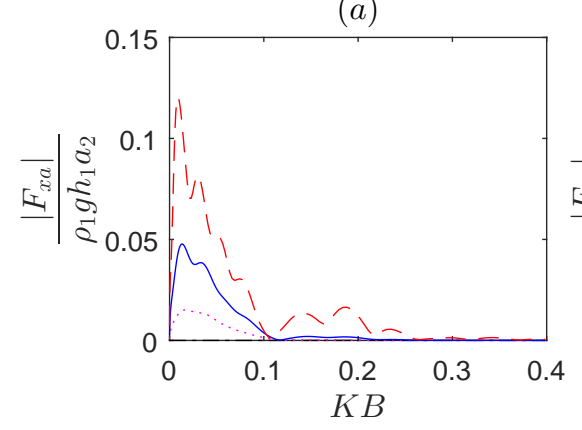

$(b)$

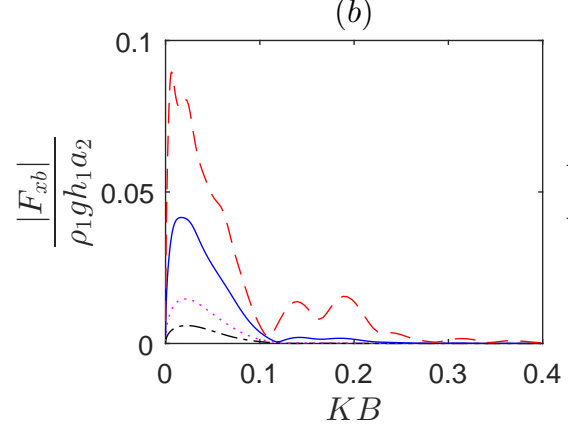

$(c)$



$(d)$

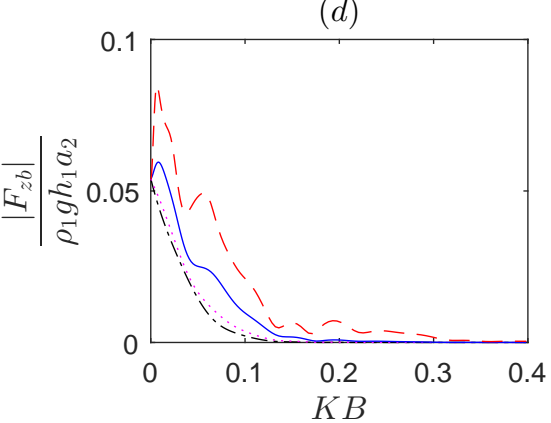

$(e)$

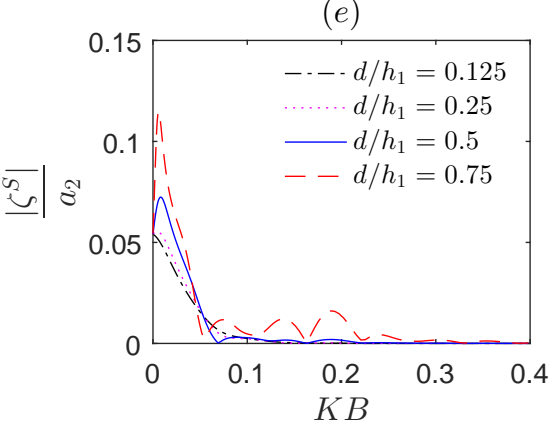

$(f)$

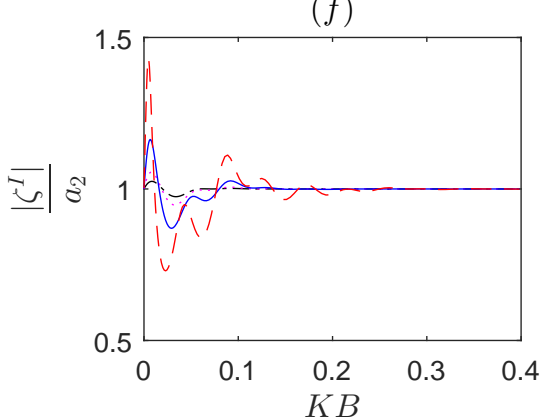

Figure 16: Wave exciting forces and wave elevations for moonpools with different $d / h_{1}$ in internal wave mode. $\gamma=0.9, h_{1} / h_{2}=1.0$, $W / B=3.0, B / h_{1}=0.5$. $(a)$ : horizontal force on Body a; $(b)$ : horizontal force on Body b; $(c)$ : vertical force on Body a; $(d)$ : vertical force on Body $\mathrm{b} ;(e)$ : surface wave elevation at WG1; $(f)$ : internal wave elevation at WG6.

mode resonance frequencies can be estimated by an approximation formula, in particular in high-frequency region.

3) In internal wave mode, as density ratio increases, the sloshing mode resonances become negligible and the frequency region where the internal wave elevation can be affected becomes narrower.

4) In surface wave mode, larger moonpool width, smaller body width or draft induces less horizontal wave exciting forces and surface wave elevation in piston mode resonance region. Smaller body width leads to less vertical wave exciting forces. In internal wave mode, as density ratio increases or depth ratio, body width or draft decreases, the maxima of wave exciting forces and wave elevations at the center of moonpool can be reduced.

5) The piston resonance frequency reduces as depth ratio decreases, but increases as moonpool width, body width or draft decreases. In addition, the sloshing mode resonance frequencies increase as moonpool width or draft decreases, while density stratification and body width have little impact on sloshing mode resonances.

\section{Acknowledgements}

We would like to thank the reviewers, who provided constructive and valuable suggestions that help to improve the paper.

\section{References}

Faltinsen, O. M., Rognebakke, O. F., Timokha, A. N., 2007. Two-dimensional resonant piston-like sloshing in a moonpool. J. Fluid Mech. 575, 359-397. 
Faltinsen, O. M., Timokha, A. N., 2015. On damping of two-dimensional piston-mode sloshing in a rectangular moonpool under forced heave motions. J. Fluid Mech. 772, R1.

Fredriksen, A. G., Kristiansen, T., Faltinsen, O. M., 2015. Wave-induced response of a floating two-dimensional body with a moonpool. Phil. Trans. R. Soc. A 373 (2033).

Jiang, S., Bai, W., Tang, G., 2018. Numerical simulation of wave resonance in the narrow gap between two non-identical boxes. Ocean. Eng. 156, 38-60.

Kristiansen, T., Faltinsen, O. M., 2008. Application of a vortex tracking method to the piston-like behaviour in a semi-entrained vertical gap. Appl. Ocean Res. 30 (1), 1-16.

Linton, C. M., McIver, M., 1995. Interaction of waves with horizontal cylinders in two-layer fluids. J. Fluid Mech. 304, 213-229.

McIver, P., 2005. Complex resonances in the water-wave problem for a floating structure. J. Fluid Mech. 536, 423-443.

Molin, B., 2001. On the piston and sloshing modes in moonpools. J. Fluid Mech. 430, 27-50.

Molin, B., Zhang, X., Huang, H., Remy, F., 2018. On natural modes in moonpools and gaps in finite depth. J. Fluid Mech. 840, 530-554.

Ning, D., Su, X., Zhao, M., Teng, B., 2015a. Hydrodynamic difference of rectangular-box systems with and without narrow gaps. J. Eng. Mech. 141 (8), 04015023.

Ning, D., Su, X., Zhao, M., Teng, B., 2015b. Numerical study of resonance induced by wave action on multiple rectangular boxes with narrow gaps. Acta Oceanol. Sin. 34 (5), 92-102.

Ning, D., Zhu, Y., C., Z., M., Z., 2018. Experimental and numerical study on wave response at the gap between two barges of different draughts. Appl. Ocean Res. 77, 14-25.

Osborne, A. R., Brown, J. R., Burch, T. L., Scarlet, R. I., 1977. The influence of internal waves on deepwater drilling operations. In: Offshore Technology Conference. Houston, Texas.

Simmons, H., Chang, M. H., Chang, Y. T., Chao, S. Y., Fringer, O., Jackson, C. R., Ko, D. S., 2011. Modeling and prediction of internal waves in the South China Sea. Oceanography 24 (4), 88-99.

Xu, W., Li, Y., Voogt, A., 2013. Internal wave soliton passage simulation during offloading. In: Proceedings of the ASME 32nd International Conference on Ocean, Offshore and Arctic Engineering. Nantes, France.

Yeung, R. W., Nguyen, T. C., 1999. Radiation and diffraction of waves in a two-layer fluid. In: Proceedings of the 22nd Symposium on Naval Hydrodynamics. Washington, D. C.

Yeung, R. W., Seah, R. K. M., 2007. On Helmholtz and higher-order resonance of twin floating bodies. J. Eng. Math. 58 (1-4), 251-265. 
Zhang, X., Bandyk, P., 2013. On two-dimensional moonpool resonance for twin bodies in a two-layer fluid. Appl. Ocean Res. 40, 1-13.

Zhang, X., Bandyk, P., 2014. Two-dimensional moonpool resonances for interface and surface-piercing twin bodies in a two-layer fluid. Appl. Ocean Res. 47, 204-218.

Zhang, X., Huang, H., Song, X., 2019. On natural frequencies and modal shapes in two-dimensional asymmetric and symmetric moonpools in finite water depth. Appl. Ocean Res. 82, 117-129.

Zhang, X., Yeung, R. W., 2016. On hydrodynamic behavior of a cylindrical moonpool with an entrapped two-layer fluid. In: Proceedings of the 31st International Workshop on Water Waves and Floating Bodies. Plymouth, Michigan, USA.

\section{Appendix A}

Expressions for $N_{j}$ :

$$
N_{j}= \begin{cases}\frac{\gamma^{2} h_{2}+\gamma\left(h_{1}-d\right)}{h-d}, & \text { for } j=0 \\ \frac{h_{2}+\sinh \left(2 \lambda_{j} h_{2}\right) / 2 \lambda_{j}}{2(h-d) \sinh ^{2} \lambda_{j} h_{2}}+\gamma \frac{h_{1}-d-\sinh \left(2 \lambda_{j}\left(d-h_{1}\right)\right) / 2 \lambda_{j}}{2(h-d) \sinh ^{2} \lambda_{j}\left(d-h_{1}\right)}, & \text { for } j=1 \\ \frac{h_{2}+\sin \left(2 \lambda_{j} h_{2}\right) / 2 \lambda_{j}}{2(h-d) \sin ^{2} \lambda_{j} h_{2}}+\gamma \frac{h_{1}-d-\sin \left(2 \lambda_{j}\left(d-h_{1}\right)\right) / 2 \lambda_{j}}{2(h-d) \sin ^{2} \lambda_{j}\left(d-h_{1}\right)}, & \text { for } j \geq 2\end{cases}
$$

Expressions for $M_{n}^{(q)}$ :

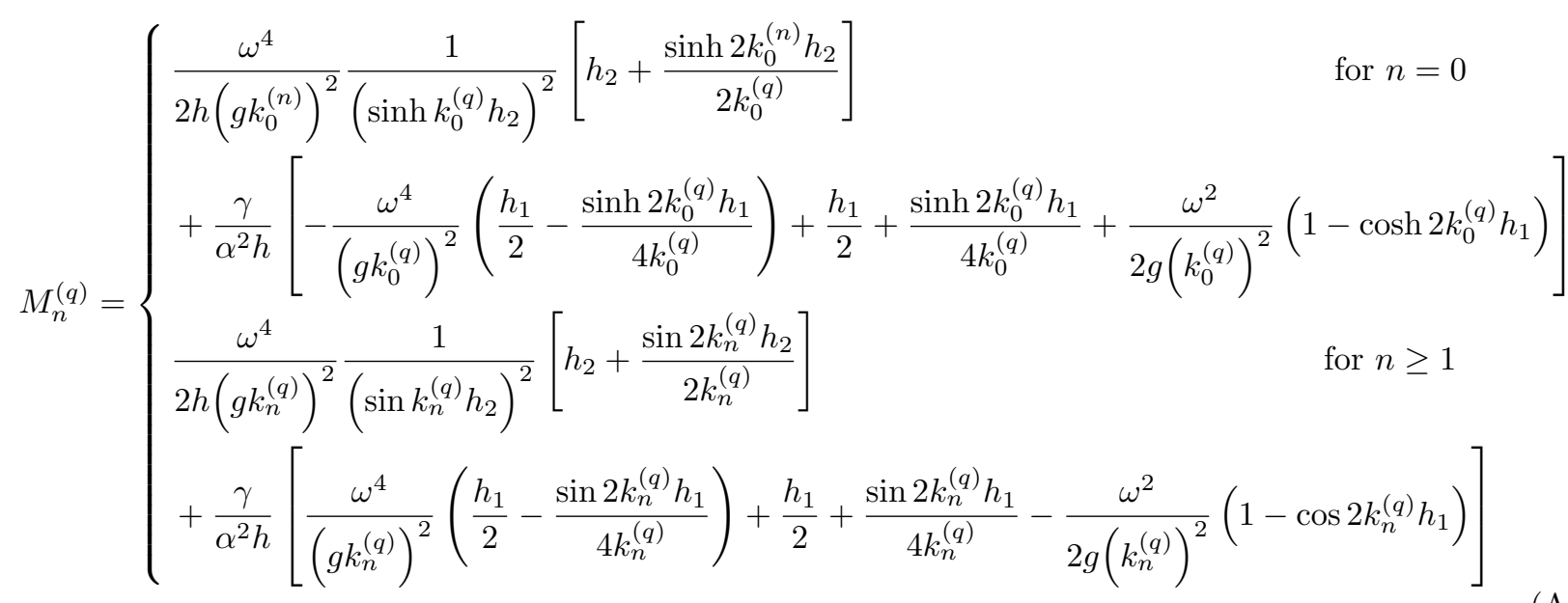

Expressions for $L_{j, n}^{(q)}$ :

For $j=0, n=0$ :

$$
L_{0,0}^{(q)}=\frac{\gamma \omega^{2}}{g\left(k_{0}^{(q)}\right)^{2}}+\frac{\gamma}{\alpha}\left[-\frac{\omega^{2}\left(\cosh k_{0}^{(q)} h_{1}-\cosh k_{0}^{(q)} d\right)}{g\left(k_{0}^{(q)}\right)^{2}}+\frac{\sinh k_{0}^{(q)} h_{1}-\sinh k_{0}^{(q)} d}{k_{0}^{(q)}}\right]
$$

For $j=0, n \geq 1$ :

$$
L_{0, n}^{(q)}=\frac{\gamma \omega^{2}}{g\left(k_{n}^{(q)}\right)^{2}}+\frac{\gamma}{\alpha}\left[\frac{\omega^{2}\left(\cos k_{n}^{(q)} h_{1}-\cos k d\right)}{g\left(k_{n}^{(q)}\right)^{2}}+\frac{\sin k_{n}^{(q)} h_{1}-\sin k_{n}^{(q)} d}{k_{n}^{(q)}}\right]
$$


For $j=1, n=0$ :

$$
\begin{aligned}
& L_{1,0}^{(q)}=\frac{\omega^{2}\left(\lambda_{1} \sinh \lambda_{1} h_{2} \cosh k_{0}^{(q)} h_{2}-k_{0}^{(q)} \cosh \lambda_{1} h_{2} \sinh k_{0}^{(q)} h_{2}\right)}{g k_{0}^{(q)}\left(\lambda_{1}{ }^{2}-\left(k_{0}^{(q)}\right)^{2}\right) \sinh \lambda_{1} h_{2} \sinh k_{0}^{(q)} h_{2}}+\frac{\gamma}{\alpha \sinh \lambda_{1}\left(d-h_{1}\right)} \\
& \cdot\left[\frac{\omega^{2}}{g k_{0}^{(q)}} \frac{\lambda_{1} \sinh \lambda_{1}\left(d-h_{1}\right) \sinh k_{0}^{(q)} h_{1}-k_{0}^{(q)}\left(\cosh k_{0}^{(q)} d-\cosh \lambda_{1}\left(d-h_{1}\right) \cosh k_{0}^{(q)} h_{1}\right)}{\lambda_{1}{ }^{2}-\left(k_{0}^{(q)}\right)^{2}}\right] \\
& +\frac{\gamma}{\alpha \sinh \lambda_{1}\left(d-h_{1}\right)}\left[\frac{-\lambda_{1} \sinh \lambda_{1}\left(d-h_{1}\right) \cosh k_{0}^{(q)} h_{1}-k_{0}^{(q)}\left(-\sinh k_{0}^{(q)} d+\cosh \lambda_{1}\left(d-h_{1}\right) \sinh k_{0}^{(q)} h_{1}\right)}{\lambda_{1}{ }^{2}-\left(k_{0}^{(q)}\right)^{2}}\right]
\end{aligned}
$$

For $j=1, n \geq 1$ :

$$
\begin{aligned}
& L_{1, n}^{(q)}=\frac{\omega^{2}\left(\lambda_{1} \sinh \lambda_{1} h_{2} \cos k_{n}^{(q)} h_{2}+k_{n}^{(q)} \cosh \lambda_{1} h_{2} \sin k_{n}^{(q)} h_{2}\right)}{g k_{n}^{(q)}\left(\lambda_{1}{ }^{2}+\left(k_{n}^{(q)}\right)^{2}\right) \sinh \lambda_{1} h_{2} \sin k_{n}^{(q)} h_{2}}+\frac{\gamma}{\alpha \sinh \lambda_{1}\left(d-h_{1}\right)} \\
& \cdot\left[\frac{\omega^{2}}{g k_{n}^{(q)}} \frac{\lambda_{1} \sinh \lambda_{1}\left(d-h_{1}\right) \sin k_{n}^{(q)} h_{1}-k_{n}^{(q)}\left(\cos k_{n}^{(q)} d-\cosh \lambda_{1}\left(d-h_{1}\right) \cos k_{n}^{(q)} h_{1}\right)}{\lambda_{1}{ }^{2}+\left(k_{n}^{(q)}\right)^{2}}\right] \\
& +\frac{\gamma}{\alpha \sinh \lambda_{1}\left(d-h_{1}\right)}\left[\frac{-\lambda_{1} \sinh \lambda_{1}\left(d-h_{1}\right) \cos k_{n}^{(q)} h_{1}+k_{n}^{(q)}\left(-\sin k_{n}^{(q)} d+\cosh \lambda_{1}\left(d-h_{1}\right) \sin k_{n}^{(q)} h_{1}\right)}{\lambda_{1}{ }^{2}+\left(k_{n}^{(q)}\right)^{2}}\right]
\end{aligned}
$$

For $j \geq 2, n=0$ :

$$
\begin{aligned}
& L_{j, 0}^{(q)}=\frac{\omega^{2}\left(\lambda_{j} \sin \lambda_{j} h_{2} \cosh k_{0}^{(q)} h_{2}+k_{0}^{(q)} \cos \lambda_{j} h_{2} \sinh k_{0}^{(q)} h_{2}\right)}{g k_{0}^{(q)}\left(\lambda_{j}^{2}+\left(k_{0}^{(q)}\right)^{2}\right) \sin \lambda_{j} h_{2} \sinh k_{0}^{(q)} h_{2}} \\
& +\frac{\gamma}{\alpha \sin \lambda_{j}\left(d-h_{1}\right)}\left[\frac{\omega^{2}}{g k_{0}(q)} \frac{\lambda_{j} \sin \lambda_{j}\left(d-h_{1}\right) \sinh k_{0}^{(q)} h_{1}+k_{0}^{(q)}\left(\cosh k_{0}^{(q)} d-\cos \lambda_{j}\left(d-h_{1}\right) \cosh k_{0}^{(q)} h_{1}\right)}{\lambda_{j}{ }^{2}+\left(k_{0}^{(q)}\right)^{2}}\right] \\
& +\frac{\gamma}{\alpha \sin \lambda_{j}\left(d-h_{1}\right)}\left[\frac{-\lambda_{j} \sin \lambda_{j}\left(d-h_{1}\right) \cosh k_{0}^{(q)} h_{1}+k_{0}^{(q)}\left(-\sinh k_{0}^{(q)} d+\cos \lambda_{j}\left(d-h_{1}\right) \sinh k_{0}^{(q)} h_{1}\right)}{\lambda_{j}{ }^{2}+\left(k_{0}^{(q)}\right)^{2}}\right]
\end{aligned}
$$

For $j \geq 2, n \geq 1$ :

$$
\begin{aligned}
& L_{j, n}^{(q)}=\frac{\omega^{2}\left(\lambda_{j} \sin \lambda_{j} h_{2} \cos k_{n}^{(q)} h_{2}-k_{n}^{(q)} \cos \lambda_{j} h_{2} \sin k_{n}^{(q)} h_{2}\right)}{g k_{n}^{(q)}\left(\lambda_{j}{ }^{2}-\left(k_{n}^{(q)}\right)^{2}\right) \sin \lambda_{j} h_{2} \sin k_{n}^{(q)} h_{2}} \\
& +\frac{\gamma}{\alpha \sin \lambda_{j}\left(d-h_{1}\right)}\left[\frac{\omega^{2}}{g k_{n}^{(q)}} \frac{\lambda_{j} \sin \lambda_{j}\left(d-h_{1}\right) \sin k_{n}^{(q)} h_{1}+k_{n}^{(q)}\left(\cos k_{n}^{(q)} d-\cos \lambda_{j}\left(d-h_{1}\right) \cos k_{n}^{(q)} h_{1}\right)}{\lambda_{j}{ }^{2}-\left(k_{n}^{(q)}\right)^{2}}\right] \\
& +\frac{\gamma}{\alpha \sin \lambda_{j}\left(d-h_{1}\right)}\left[\frac{-\lambda_{j} \sin \lambda_{j}\left(d-h_{1}\right) \cos k_{n}^{(q)} h_{1}-k_{n}^{(q)}\left(-\sin k_{n}^{(q)} d+\cos \lambda_{j}\left(d-h_{1}\right) \sin k_{n}^{(q)} h_{1}\right)}{\lambda_{j}{ }^{2}-\left(k_{n}^{(q)}\right)^{2}}\right]
\end{aligned}
$$

